Titre : Réticence à la vaccination et recours aux médecines alternatives et complémentaires

Auteur : Eve Dubé, Chantal Sauvageau et Dominique Gagnon

Publication : La santé publique à une ère marquée par le doute - Origines religieuses et culturelles de l'hésitation des Canadiens face à la vaccination

Directeurs : Paul Bramadat, Maryse Guay, Julie A. Bettinger et Réal Roy

Pages : $174-204$

ISBN : 978-2-7622-0359-2

URI : http://hdl.handle.net/11143/16022

DOI : https://doi.org/10.17118/11143/16022

Éditeur : Les Éditions de l'Université de Sherbrooke (ÉDUS) 


\title{
Réticence à la vaccination et recours aux médecines alternatives et complémentaires
}

\author{
EVE DUBÉ, CHANTAL SAUVAGEAU ET DOMINIQUE GAGNON
} "Devant une information qui contredit nos croyances personnelles... c'est elle, et non
pas nous-mêmes, que nous mettons en doute. "

- Eula Biss, On Immunity: An Inoculation (2014)

Le temps où les responsables de la santé pouvaient publier des recommandations fondées sur les meilleures données médicales et scientifiques disponibles et s'attendre à ce qu'elles soient respectées est peut-être révolu.

- Margaret Chan, directeur général de l'OMS, Rapport du Directeur général au Conseil exécutif à sa cent vingt-sixième session (cité dans Larson et Schulz, 2015)

\section{Introduction}

Chez l'homme, toutes les cultures se sont dotées de systèmes médicaux. Ces sytèmes sont décrits largement comme un ensemble de techniques et de pratiques ayant pour but de guérir les maladies et de maintenir la santé. Ces systèmes ont des caractéristiques communes (notamment les méthodes utilisées dans la normalisation de la santé et le diagnostic des maladies, les rôles et leur définition, ainsi que les carrières offertes aux prestataires de soins). Cependant, chaque systèmes'inscrit dans un contexte historique et sociopolitique particulier (Kleinman, 1997). Jusqu'au XIXe siècle, la médecine nord-américaine se caractérisait par l'offre de plusieurs options en matière de soins de santé, maisaucun ordre professionnel ne distinguait les praticiens (Kaptchuk et Eisenberg, 2001). En plus des chirurgiens, des barbiers et des apothicaires, les soins de santé étaient offerts par des herboristes et des sagesfemmes, (Kaptchuk et Eisenberg, 2001). Les rares médecins ayant été formés à l'université réservaient leurs services à la classe privilégiée (Kaptchuk et Eisenberg, 2001). À compter du début du XXe siècle, la biomédecine, aussi appelée la médecine allopathique ou la médecine occidentale traditionnelle, devint graduellement le modèle dominant. L'émergence de la biomédecine est associée à la découverte de « preuves » de la maladie, grâce aux progrès de 
l'anatomopathologie, de la dissection du corps humain et de la microbiologie (Pélissier-Simard et Xhignesse, 2008; Kleinman, 1997). En dépit de l'existence de différentes formes de médecine et de méthodes de guérison qui se sont fait une place aux dépens de la nouvelle orthodoxie médicale (notamment l'hydropathie et l'homéopathie), la biomédecine a graduellement acquis une légitimité et une hégémonie grâce aux lois régissant l'enseignement et la pratique de la médecine (Kaptchuk et Eisenberg, 2001). Comme l'indiquent Bramadat, Brunk et Roy, respectivement aux chapitres 1, 3 et 4 du présent ouvrage, l'information générée et utilisée par les chercheurs en biomédecine est présentée comme étant objective, neutre et universelle. La biomédecine s'intéresse aux anomalies de la structure ou du fonctionnement des organes et du système physiologique; sa cible principale est la « maladie». Celle-ci comporte d'autres aspects, notamment l'expérience personnelle et subjective de la souffrance (ne pas se «sentir bien ») ou le concept socioculturel associé à des états de santé dévalorisés (être " malade»), mais ils sont généralement, dans le discours biomédical, subordonnés à la définition mécaniste dominante (Kleinman, 1997; Massé, 1995).

Il faudra attendre les années 1960 et la contre-culture pour que les pratiques de santé parallèles comme l'homéopathie et la naturopathie redeviennent populaires. Dans les grands centres urbains, on s'adonna de plus en plus à la méditation, au yoga et à la visualisation. Le cri de ralliement de l'heure était de «reprendre le contrôle de son corps » et l'on dénonçait la "médicalisation » et le paternalisme. Les mouvements féministes, par exemple, s'opposaient au paternalisme inhérent à la relation thérapeutique établie par les professionnels de santé biomédicaux (Kaptchuk et Eisenberg, 2001).

Nous tenterons, dans le présent chapitre, de rendre compte de ces différentes tendances à l'échelle canadienne et de favoriser une meilleure compréhension des approches parallèles en matière de soins de santé. Après avoir donné une définition générale de celles-ci, nous nous intéresserons plus particulièrement à quatre d'entre elles, soit la naturopathie, l'homéopathie, la chiropratique et la pratique sage-femme, sélectionnées non pas parce qu'elles sont les mieux connues et les plus populaires au Canada, mais parce qu'elles sont souvent associées à une position assez bien définie au sujet de la vaccination. Nous terminerons sur une description des influences que pourraient avoir ces approches sur le phénomène de l'hésitation à l'égard de la vaccination dans la population en général. En examinant de plus près la relation entre l'intérêt croissant pour les approches complémentaires et parallèles et la hausse simultanée de la réticence à la vaccination, nous pourrons mieux illustrer comment se manifestent d'importants changements culturels - liés aux crises de la confiance et de la vérité.

\section{Médecine traditionnelle et approches complémentaires, alternatives et parallèles : quelques définitions}

S'il se trouve un consensus sur les pratiques médicales alternatives, c'est justement que l'on ne s'entend pas sur leur nature et leur portée. Dans les années 1970 et 1980, on parlait principalement d'approches « holistiques » et de médecine « naturelle » (Pélissier-Simard et Xhignesse, 2008). L'expression « médecines alternatives et complémentaires » (MAC) semble être aujourd'hui celle que l'on emploie le plus souvent pour regrouper ces pratiques et cultures hétérodoxes (Wieland, Manheimer et Berman, 2011). Cependant, malgré sa présence fréquente dans les articles universitaires et la presse populaire, tous ne s'entendent pas sur la 
pertinence de ces termes. Certains estiment que l'expression « approches complémentaires et alternatives » serait préférable, puisqu'on y inclut des démarches qui ne sont pas proprement médicales (Santé Canada, 2001). Pour d'autres, c'est le choix du qualificatif « alternatives » qui pose problème, car il suppose une substitution; ceux-là proposent plutôt l'expression « approches complémentaires et parallèles » (Santé Canada, 2001). Aux États-Unis, le National Center for Complementary and Alternative Medicine (maintenant appelé le National Center for Complementary and Integrative Health), qui fait partie des National Institutes of Health, recommandait depuis plusieurs années l'utilisation de l'expression " médecine complémentaire et alternative », mais a révisé sa position en juillet 2014 et utilise maintenant " approches complémentaires en santé » lorsqu'il est question de pratiques et produits convenant à différentes conditions de santé (NIH, 2016). Même si certains nous en feront reproche, nous emploierons l'expression " médecines alternatives et complémentaires » et l'abréviation MAC, car ce sont les plus fréquemment utilisées dans la littérature actuelle'. II est aussi difficile de choisir un terme approprié pour représenter la médecine occidentale; cette dernière pouvant être désignée de différentes façons telles que, comme la biomédecine «traditionnelle », la médecine « allopathique » ou la médecine « scientifique ». Nous avons choisi, pour ce chapitre, le terme le plus commode, à savoir la biomédecine.

C'est une tâche complexe que de cerner la vaste et constamment évolutive portée des MAC et la frontière qui les sépare de la biomédecine. On définit souvent les MAC par ce qu'elles ne sont pas: on ne les enseigne pas à l'université, elles ne fonctionnent pas selon le paradigme des concepts biomédicaux, on ne les applique pas dans les hôpitaux généraux, elles ne sont pas prouvées scientifiquement, et ainsi de suite (Ernst, Cohen et Stone, 2004). Parmi les définitions que l'on utilise couramment figure la suivante: Les médecines alternatives et complémentaires représentent un vaste ensemble de ressources comprenant notamment des systèmes de santé, leurs modalités et pratiques, ainsi que les théories et convictions qui y sont associées, mais qui ne sont pas celles du système dominant, dans une société ou une culture et à une période données. Les MAC comportent des ressources que leurs utilisateurs perçoivent comme étant associées à des résultats positifs sur le plan de la santé. II n'y a pas toujours de limites claires ou fixes entre les champs couverts par les MAC et ceux du système dominant (Institute of Medicine [US] Committee on the Use of Complementary and Alternative Medicine by the American Public, 2005).

Ajoutons à cela qu'une perspective aussi large n'est guère utile au quotidien (Wieland, Manheimer et Berman, 2011). On a en effet recensé, parmi les MAC, jusqu'à quatre mille pratiques ou disciplines différentes (Pélissier-Simard et Xhignesse, 2008). Certaines de ces approches composent un système de santé complet, allant du diagnostic au traitement - la médecine chinoise traditionnelle en est un exemple - tandis que d'autres se concentrent que sur certains éléments spécifiques liés au traitement ou à la prévention- par exemple, le recours aux suppléments alimentaires ou à des programmes de perte de poids ou de conditionnement physique commerciaux ou à des groupes de soutien comme les Alcooliques Anonymes (Santé Canada, 2001).

1. De manière générale, le terme « complémentaire » renvoie aux situations dans lesquelles on a recours à des approches non orthodoxes en même temps qu'à la médecine conventionnelle, tandis que le terme " alternative " désigne le recours aux approches non orthodoxes uniquement, à l'exclusion de la médecine conventionnelle. 
Produits naturels

Comprend une large gamme de produits, comme les plantes médicinales, les vitamines, les minéraux et les probiotiques

Pratiques visant le corps et l'esprit

Comprend une vaste gamme diversifiée de procédures ou de techniques qu'un enseignant ou un praticien compétent exécute ou montre comment exécuter.

Exemples: acupuncture, massothérapie, méditation, thérapies par le mouvement comme la méthode Pilates et le rolfing, techniques de relaxation comme l'imagerie guidée et les exercices de respiration, la manipulation vertébrale, le qi gong, le taichi, le yoga, le toucher thérapeutique et l'hypnothérapie

Autres approches complémentaires en santé

Les deux premières catégories (produits naturels et pratiques visant le corps et l'esprit) rassemblent à elles seules la plupart des approches complémentaires. Les autres ne correspondent ni à l'une, ni à l'autre.

Exemples: médecine chinoise traditionnelle, médecine ayurvédique, guérisseurs traditionnels, homéopathie, naturopathie

Source : https://nccih.nih.gov/health/integrative-health

On pourra trouver d'autres définitions des MAC dans les systèmes de classification conçus pour organiser des approches et des pratiques en catégories différentes. L'un des organismes composant les National Institutes of Health des États-Unis, le National Center for Complementary and Integrative Health $(\mathrm{NCClH})$, dont le rôle consiste à financer la recherche sur les approches complémentaires et parallèles en santé, préconise une classification fondée sur les catégories générales suivantes : produits naturels, pratiques visant le corps et l'esprit et autres approches complémentaires en santé (voir le tableau 7.1).

Cette classification comporte certains inconvénients car, en raison de la définition non formelle et de la large portée des catégories, certaines pratiques peuvent relever de plusieurs d'entre elles. Tataryn (2002) propose quatre paradigmes pouvant regrouper les différentes approches et pratiques reliées à la santé et à la maladie (se reporter au tableau 7.2). Le premier paradigme est celui du « corps »; il regroupe les thérapies qui «ciblent les facteurs biologiques comme les déterminants primaires de la santé et de la maladie » (877). Le paradigme «corpsesprit » souligne l'influence du cerveau sur la santé et la maladie. Le troisième paradigme, appelé "corps-énergie », rassemble les thérapies reposant sur l'hypothèse que «la vie, voire l'univers entier, repose sur l'équilibre, le mouvement et l'interaction d'énergies subtiles » (885). Le paradigme « corps-spiritualité » occupe le quatrième rang et rappelle « l'existence d'un ou de plusieurs êtres ou états de conscience de nature supra locale et non physique, qui transcendent l'univers matériel, mais peuvent agir sur lui. Parmi ces êtres figurent Dieu, Jéhovah, Tao, Bouddha, Atma, la Source, les anges, les esprits etou d'autres êtres supérieurs. » (886) Tataryn (2002) est d'avis que ces paradigmes sont hiérarchisés, chacun acceptant les hypothèses des précédents. Le paradigme corps-esprit, par exemple, considère que le corps, l'esprit, l'énergie et la spiritualité sont autant de dimensions de la santé et de la maladie. Ce système de classement mérite l'attention, car il ne crée pas de dichotomie entre la biomédecine et les MAC. Au contraire, il propose une approche qui classe les pratiques selon leurs hypothèses sous-jacentes au sujet de la nature de la santé et de la maladie et selon leurs thérapies et approches préconisées. On constate un chevauchement entre les différentes formes des MAC comme entre leurs partisans; il s'exprime dans la relation complexe que font les personnes qui décrivent leurs motifs de réticence à la vaccination. Il est en effet difficile de déterminer si l'hésitation que l'on éprouve trouve son origine dans une résistance vis-à-vis un aspect particulier du paradigme dominant du « corps » ou dans une attirance vers des forces invisibles (spirituelles, émotionnelles, intuitives, etc.). 
Tableau 7.2 Classification des thérapies usuelles selon les quatre paradigmes de la santé et de la maladie de Tataryn

\begin{tabular}{|l|l|}
\hline Corps & $\begin{array}{l}\text { 1. Substances physiques } \\
\text { - Régimes et suppléments } \\
\text { Aromathérapie, régime de Gerson, plantes médicinales, régime macrobiotique, thérapies minérales et } \\
\text { vitaminiques } \\
\cdot \text { Extraits et concentrés } \\
\text { Antinéoplastons, thérapies cellulaires, laetrile, ozonothérapie, cartilage de requin } \\
\text {. Produits chimiques/synthétiques } \\
\text { Chimiothérapie, 714-X, thérapie par chélation } \\
\text { 2.Manipulation physique } \\
\text { Massage, physiothérapie, chiropratique, lavements, irrigation du colon, thérapie hypo/hyperthermique, } \\
\text { radiothérapie, chirurgie }\end{array}$ \\
\hline Corps-esprit & $\begin{array}{l}\text { Affirmations/suggestion, counseling, interprétation des rêves, thérapies par l'art, hypnose, imagerie/visuali- } \\
\text { sation, méditation, psychothérapie, réduction du stress, groupes de soutien }\end{array}$ \\
\hline Corps-énergie & $\begin{array}{l}\text { Digitopuncture, acupuncture, médecine ayurvédique, médecine chinoise, thérapie par les cristaux, ho- } \\
\text { méopathie, thérapie magnétique, thérapie par polarité, qi gong, réflexologie, reiki, toucher thérapeutique, } \\
\text { tai-chi, yoga }\end{array}$ \\
\hline Corps-spiritualité & $\begin{array}{l}\text { Cérémonies et rites, danse derviche, exorcisme, guérison par la foi, traditions autochtones, imposition des } \\
\text { mains, magie / pratiques occultes, prière, diagnostic psychique, interventions psychiques, sacrements/rites, } \\
\text { guérison chamanique }\end{array}$ \\
\hline
\end{tabular}

Source : Tataryn (2002)

Comme le laissent entrevoir les tableaux 7.1 et 7.2, les MAC englobent de multiples pratiques. II ne faut pas tenir pour acquis qu'il existe une authentique dichotomie entre elles et la biomédecine (Fadlon, 2005; Ning, 2013). Fadlon (2005), par exemple, qui a réalisé de nombreux travaux dans des villes israéliennes, explique la popularité croissante des MAC (qu'elle appelle la « médecine non conventionnelle ») par un processus de " domestication ». Les succès des MAC pourraient ainsi être attribués à sa fusion avec la biomédecine, "créant ainsi une forme hybride de traitement médical suffisamment étrange pour être fascinant, mais tout de même suffisamment familier pour ne pas être déconcertant » (7).

Dans le reste du présent chapitre, nous examinerons plus en profondeur quatre formes de MAC : la naturopathie, l'homéopathie, la chiropratique et la pratique sage-femme. Bien que I'homéopathie soit une approche thérapeutique utilisée en naturopathie, nous lui accorderons une attention particulière, car elle figure parmi les approches naturopathiques les plus populaires. Notons également que la réticence des homéopathes, en matière de vaccination, et l'influence qu'elle peut avoir sur leur clientèle comptent parmi les inquiétudes les plus fréquentes des professionnels de la santé issus de la biomédecine (Société canadienne de pédiatrie, 2005; Ernst, 2001; Weeks, 2015a, 2015b; Picard, 2015). De même, la pratique sage-femme est financée par les fonds publics et pratiquée dans des établissements de biomédecine dans dix provinces et territoires canadiens qui la réglementent. En ce sens, cela ne correspond pas à la plupart des définitions des MAC, à savoir qu'il s'agit de « pratiques médicales pour l'instant non admises dans le cadre de systèmes de soins de santé orthodoxes régis par l'État » (Ning, 2013, 2). Bien que certains spécialistes se basent sur ce motif pour ne pas englober la pratique sage-femme dans les MAC, nous avons choisi de l'inclure parce qu'il existe des données probantes à l'effet que les enfants nés avec l'aide d'une sage-femme sont moins susceptibles d'être immunisés, comparativement aux autres (Boulianne et coll., 2011; Guay et coll., 2009; Zhang et coll., 2008; Boulianne et coll., 2013). Par ailleurs, cette pratique, encore non réglementée au Canada dans les années 1990, est apparue de manière marginale 
dans la biomédecine à la demande de groupes de femmes contestant la « médicalisation » de la grossesse et de l'accouchement et réclamant une réappropriation de la maternité (Gagnon, 2012). En fait, certaines sages-femmes s'interrogent ouvertement au sujet des normes et des pratiques de la biomédecine. De plus, comme l'indiquent les résultats d'une récente revue de la littérature, le recours aux thérapies préconisées par les MAC, telles que les plantes médicinales, l'acupuncture et l'aromathérapie, est très répandu dans la pratique sage-femme (Hall, McKenna et Griffiths, 2012).

Outre le fait qu'une vaste littérautre décrit la relation entre ces formes de MAC et la réticence à la vaccination (se reporter au chapitre 6 du présent ouvrage, par Guay, Dubé et Laberge), nous avons choisi d'étudier ces approches parce qu'elles comptent parmi les plus populaires au Canada. En effet, selon les plus récentes données nationales de l'Enquête sur la santé dans les collectivités canadiennes de 2003, 20 p. cent des Canadiens âgés de 12 ans ou plus, soit 5,4 millions de personnes, ont déclaré avoir obtenu des soins de santé non conventionnels ou complémentaires au cours de l'année précédente, ce qui représente une hausse marquée par rapport aux données de 1994-1995, où la proportion de Canadiens âgés de 18 ans ou plus ayant reçu de tels soins n'était que de 15 p. cent (Park, 2005). Les professionnels les plus fréquemment consultés étaient les chiropraticiens (avec 11 p. cent des répondants), contre 2 p. cent pour ceux ayant vu un homéopathe ou un naturopathe (Park, 2005). Par ailleurs, en 2006-2007, au Canada, 6,1 p. cent des naissances se sont faites sous la surveillance d'une sage-femme ("Ce que disent les mères », 2009). Il est difficile de décrire les caractéristiques des utilisateurs de MAC au Canada. En dépit du fait que les facteurs socioculturels ayant une incidence sur le recours aux MAC soient encore mal compris, les études montrent généralement que les femmes d'âge moyen, scolarisées, mais ayant une moins bonne santé les utilisent plus que d'autres (Harris et coll., 2012). Cette situation pourrait cependant changer, car des chercheurs canadiens ont indiqué que le recours aux MAC « risque de ne plus être un phénomène limité aux personnes davantage scolarisées et mieux nanties » (Andrews et Boon, 2005, 22).

Pour chaque type de MAC sélectionné, nous décrirons les pratiques et approches communes, la perception de la santé et de la maladie qui y est associée et la portée de l'institutionnalisation au Canada ${ }^{2}$. Ces renseignements proviennent principalement du site Web des pincipales associations professionnelles canadiennes. Le lecteur trouvera donc dans ces pages l'opinion des praticiens eux-mêmes et non celle des autorités de la santé publique ou de chercheurs indépendants.

2. Au Canada, les produits liés à la santé sont réglementés par le gouvernement fédéral et les pratiques en soins de santé relèvent des autorités provinciales. Les soins de santé sont pour la plupart prodigués à même les fonds publics, mais l'émergence d'une demande nouvelle a encouragé l'expansion de secteurs relevant de l'entreprise privée, comme les MAC. La pratique des MAC est réglementée individuellement par les provinces et territoires canadiens, ce qui crée une certaine diversité quant à savoir qui est assujetti et dans quelle mesure. Les praticiens qui exercent les MAC dans des territoires où elles ne sont pas réglementées ont toute liberté d'action, à condition de ne pas contrevenir aux autres lois (Andrews et Boon, 2005). En effet, les MAC ne sont pas financées par les gouvernements et leur accès n'est pas universel, comme la biomédecine; la plupart des Canadiens doivent payer de leur poche les honoraires des praticiens et les produits que ces derniers prescrivent. 


\title{
Naturopathie, homéopathie, chiropratique et pratique sage-femme: vue d'ensemble de la situation canadienne
}

\author{
Naturopathie
}

Il est difficile de cerner avec précision les origines de la naturopathie, car cette approche comprend des systèmes de croyance ayant évolué depuis l'Antiquité, dans différentes parties du monde (Busse, Wilson et Campbell, 2008). La popularité croissante de la naturopathie dans les pays industrialisés pourrait être associée au mouvement du nouvel âge, avec lequel elle a de nombreuses caractéristiques communes - dont une approche éclectique et individualisée en matière de spiritualité. Dans son site Web, l'Association canadienne des docteurs en naturopathie définit la naturopathie de cette façon : "La naturopathie est un système de soins de santé primaires distinct qui allie les connaissances scientifiques modernes aux formes traditionnelles et naturelles de la médecine. » (ACDN, 2017c) Selon cette association, la naturopathie englobe l'art et la science du diagnostic, du traitement et de la prévention des maladies au moyen de thérapies naturelles combinant les plantes médicinales, la nutrition clinique, l'hydrothérapie, l'homéopathie, les manipulations naturopathiques, la médecine chinoise traditionnelle et l'acupuncture ainsi que la création d'une forte relation thérapeutique entre le patient et le praticien. La naturopathie cherche à améliorer la santé et prévenir les maladies en suggérant de nouvelles habitudes de vie et certains changements d'attitudes. Son objectif n'est pas simplement de traiter la maladie, mais aussi de détoxifier et de revigorer le corps. L'approche naturopathique valorise la personne qui assume la responsabilité de sa santé pour promouvoir un bien-être optimal grâce à des méthodes naturelles qui sont sans danger pour l'environnement. Les traitements sont sélectionnés en fonction du patient " entier ", en supposant que "la maladie affecte toute la personne et non simplement un organe ou un système » (ACDN, 2017a). Le docteur en naturopathie cherche à traiter la cause et non les symptômes de tout état et prend en considération non seulement les signes physiques, mais aussi les facteurs mentaux, émotionnels, génétiques, environnementaux, sociaux, spirituels et autres (ACDN, 2017a).

Selon l'ACDN, au-delà de 2400 docteurs en naturopathie sont à l'œuvre au Canada (ACDN, 2017b). Cinq provinces (Colombie-Britannique, Alberta, Saskatchewan, Manitoba et Ontario) réglementent la pratique de la naturopathie. Elles exigent toutes l'obtention d'un permis d'exercice. En Nouvelle-Écosse, la loi intitulée « Naturopathic Doctors Act » de 2008 protège le titre des docteurs en naturopathie (ACDN, 2017b). Au Canada, les seuls établissements d'enseignement agréés par le Council on Naturopathic Medical Education (CNME) sont le Canadian College of Naturopathic Medicine (CCNM), situé en Ontario, et le Boucher Institute of Naturopathic Medicine (BINM), en Colombie-Britannique. Dans les territoires où la pratique de la naturopathie n'est pas réglementée, le contenu des cours varie selon les établissements et les organismes. Certains n'offrent qu'une formation minimale, mais d'autres proposent un programme complet, similaire à celui que l'on retrouve dans les établissements agréés et comprenant des cours de biologie, de chimie et de médecine, ainsi que des stages et des examens standardisés. 


\section{Homéopathie}

L'homéopathie est une approche thérapeutique couramment offerte dans un cadre naturopathique (ACDN, 2017c), mais elle est également proposée par des cliniciens en homéopathie. Les homéopathes diagnostiquent et traitent les maladies en administrant des produits homéopathiques (minuscules doses chimiques ou naturelles de la maladie elle-même qui produiront un des symptômes généralement associés à celle-ci) ou en recommandant des changements dans le mode devie (Ressources humaines et Développement des compétences Canada, 2017c). Le médecin et chimiste allemand Samuel Hahnemann (1755-1843) a été le premier à proposer cette approche. L'homéopathie s'appuie sur deux grands principes : la loi de similitude et le principe des hautes dilutions. La loi de similitude est basée sur le concept voulant que "le semblable guérit son semblable». Ce concept, attribué à Hippocrate, laisse supposer qu'une substance provoquant un ensemble de symptômes chez une personne en santé pourra également guérir un malade présentant ces mêmes symptômes. Le mot " homéopathie » découle de ce principe car, en grec, « homéo » signifie similaire et " pathos » signifie maladie ou souffrance. Le processus de traitement homéopathique comprend donc, parmi ses éléments essentiels, le choix du remède en fonction des symptômes plutôt que de la maladie (Société canadienne de pédiatrie, 2005). Cette approche est donc contraire à la biomédecine, qui cherche à « lutter » contre la maladie et à éliminer les symptômes (Ernst, 2015a). La théorie homéopathique prétend également que la dilution du remède peut en accroître le pouvoir curatif. Par conséquent, plus grand est le nombre de dilutions (et moins grande est la présence de la substance originale dans la solution), plus puissant sera le remède. De plus, après chaque dilution, le remède est fortement agité ou secoué (ce que l'on appelle des "succussions») afin de le "dynamiser » et d'en révéler le pouvoir "caché ». Le processus de dynamisation par succussion est jugé essentiel à l'efficacité et à la " puissance » du produit. En homéopathie, les dilutions en série (que l'on appelle la potentialisation) vont parfois si loin qu'à la fin, il ne reste plus de molécules de la substance originale dans le produit dilué. Hahnemann est à l'origine de la dilution centésimale (ou dilution $\mathrm{CH}$ ), qui signifie que la substance est diluée au centième à chaque étape. Il suggérait qu'une dilution à $30 \mathrm{CH}$ pouvait convenir dans la plupart des cas (pour un facteur de dilution de 10-60).

Au Canada, tous les produits issus des MAC sont réglementés par le gouvernement fédéral (Andrews et Boon, 2005). Depuis janvier 2004, les produits homéopathiques homologués doivent aussi porter un numéro d'identification de médicament homéopathique (DIN-HM), qui leur est attribué par la Direction des produits de santé naturels et sans ordonnance de Santé Canada. La réglementation a pour objectif, selon le site Web de Santé Canada, « de [s'assurer] que tous les Canadiens ont facilement accès à des produits de santé naturels sûrs, efficaces et de grande qualité » (Santé Canada, 2017; italiques ajoutés par les auteurs). De nombreux produits homéopathiques peuvent néanmoins être achetés sans ordonnance et sans avis du médecin. Par ailleurs, les homéopathes sont fort différents, du point de vue de leur formation et de leur professionnalisation : certains n'ont suivi aucun cours, tandis que d'autres ont obtenu un diplôme au bout d'une formation continue de quatre ans, au Canada ou ailleurs dans le monde. Au Canada, la National United Professional Association of Trained Homeopaths (NUPATH) exige que ses membres suivant une formation standardisée minimale avant de leur décerner une attestation et le titre de praticiens agréés en homéopathie (R.Hom.) ou de docteurs en naturopathie (HD) (NUPATH, 2015). II existe plusieurs autres associations professionnelles représentant les homéopathes au Canada. Il est difficile d'apprécier le rôle de 
chacune, car elles se distinguent, dans la pratique, sur le plan des critères d'admissibilité des membres, de leurs fonctions et de leurs codes de conduite. En fait, l'estimation du nombre d'homéopathes, au Canada, est une tâche très ardue. II n'y a pas de liens évidents entre les organismes professionnels et un seul praticien pourrait fort bien être membre de plusieurs d'entre eux. En outre, l'homéopathie est généralement pratiquée comme complément à une autre profession de la santé (Société canadienne de pédiatrie, 2005). Pour terminer, l'Ontario est devenue, le $1^{\text {er }}$ avril 2015, la première province canadienne à réglementer la pratique de l'homéopathie, avec l'adoption de la Loi sur les homéopathes (2007).

\section{Chiropratique}

La chiropratique est une méthode mise au point à la fin du XIXe siècle par D.D. Palmer, qui a mis de l'avant une nouvelle théorie de la maladie centrée sur les lésions du système nerveux, qu'il appelait des subluxations. À l'origine, la théorie de la chiropratique découlait du principe voulant que la plupart, sinon la totalité des maladies étaient attribuables à des lésions ou à des dysfonctionnements des articulations (Busse, Morgan et Campbell, 2005).

Les chiropraticiens diagnostiquent, traitent et préviennent les troubles neuromusculosquelettiques liés à la colonne vertébrale, au système nerveux, au bassin et aux autres articulations du corps, en redressant la colonne ou en effectuant d'autres manipulations correctives (Ressources humaines et Développement des compétences Canada, 2017a). Ils soignent les troubles de la santé en ayant recours à des méthodes naturelles, par exemple des rectifications ou des manipulations des articulations, et par des traitements complémentaires tels que l'électrothérapie, l'acupuncture, la thermothérapie et les massages, en prêtant une attention particulière à la colonne vertébrale (Ressources humaines et Développement des compétences Canada, 2017a).

L'Association chiropratique canadienne (ACC) défend les intérêts professionnels des chiropraticiens canadiens. Selon cette association, «la chiropratique est un traitement non effractif et non pharmacologique qui fortifie le système musculosquelettique (MS) et les fonctions neurologiques connexes grâce à une thérapie manuelle globale et éprouvée (ACC, 2015b). Toujours selon l'association, il y a en ce moment plus de 8400 chiropraticiens agréés au Canada.

La formation en chiropratique comprend au moins deux années d'études postsecondaires en sciences, suivies d'un programme de quatre ou cinq ans d'études en chiropratique dispensé par un établissement reconnu par la commission d'accréditation du Council on Chiropractic Education; il faut ensuite réussir les examens du Conseil canadien des examens chiropratiques et ceux de l'organisme provincial responsable d'accorder les permis (Ressources humaines et Développement des compétences Canada, 2017a). La formation en chiropratique peut être obtenue dans deux établissements canadiens : le Canadian Memorial Chiropractic College (CMCC), à Toronto, et I'Université du Québec à Trois-Rivières, au Québec. Les candidats au doctorat en chiropratique (DC) doivent réussir les examens du Conseil canadien des examens chiropratiques, ainsi que ceux qui sont administrés par les autorités provinciales (ACC, 2015c).

Toutes les provinces canadiennes et le territoire du Yukon ont adopté des lois régissant la 
chiropratique. Dans chaque province, des associations professionnelles supervisent la pratique et délivrent des permis d'exercice aux praticiens ayant terminé la formation obligatoire (ACC, 2015c). La portée des services de chiropratique, dans toutes les provinces, englobe le diagnostic, la prescription et l'interprétation de radiographies ainsi que la manipulation de la colonne vertébrale. Certaines provinces permettent aussi au chiropraticien de recommander un patient à un médecin spécialiste (Ressources humaines et Développement des compétences Canada, 2017a).

\section{Pratique sage-femme}

Les sages-femmes sont des professionnelles des soins de santé primaires qui exercent dans différents milieux (à domicile, dans la collectivité, à l'hôpital, en clinique ou en unité de santé, etc.). L'Association canadienne des sages-femmes (2015b) décrit ainsi le rôle de la sage-femme:

Les sages-femmes offrent tous les soins requis pour une grossesse à faible risque, avant l'accouchement, pendant le travail et après la naissance; ces soins comprennent les examens physiques, les tests de dépistage et de diagnostic, l'évaluation des risques et des conditions anormales et la supervision d'accouchements par voie vaginale normaux. Les sages-femmes travaillent en collaboration avec d'autres professionnels de la santé et recherchent l'avis de médecins spécialistes ou dirigent leurs clientes vers ceux-ci, selon les besoins. Le modèle de pratique sage-femme fait la promotion d'une naissance normale, habilite la femme pour qu'elle fasse des choix avertis et assure la continuité des soins et du soutien tout au long de la grossesse. Les sages-femmes assistent aux accouchements en milieu hospitalier, dans des centres de naissance et à domicile.

La pratique sage-femme considère que la grossesse et l'accouchement sont des processus physiologiques normaux qui méritent le respect. Les sages-femmes veillent à protéger la santé des femmes, des nourrissons et des familles, en tenant compte des aspects sociaux, émotionnels, culturels et physiques de l'expérience maternelle et des besoins féminins. Leurs tâches comprennent l'éducation prénatale et postnatale et peuvent porter sur d'autres aspects de la santé reproductive, le rôle des parents ou les soins aux enfants (CCOSF, 2017). Selon le site de leur association, les sages-femmes canadiennes respectent le droit des femmes de faire des choix éclairés pour toutes les questions liés à leur santé et à leurs soins. Elles encouragent leurs patientes à prendre des décisions éclairéesen leur fournissant une information complète, pertinente et objective dans le cadre d'une relation personnelle d'égale à égale. Les sagesfemmes canadiennes doivent s'informer des plus récentes recherches au sujet de questions relatives à la maternité et s'assurer d'être en mesure d'en faire une analyse critique en vue d'intégrer à leur pratique tous les résultats pertinents (CCOSF, 2017). 
La profession de sage-femme est réglementée dans la plupart des provinces et territoires canadiens (à l'exception de Terre-Neuve-et-Labrador, de l'île-du-Prince-Édouard, du NouveauBrunswick et du Yukon). Bien que les lois des provinces et des territoires diffèrent quelque peu, tous ceux qui réglementent la profession ont adopté une structure et un modèle de pratique semblables pour guider la tâche des sages-femmes (CCOSF, 2017).

Pour devenir sage-femme, il faut avoir obtenu un diplôme universitaire de premier cycle en pratique sage-femme, ou l'équivalent, et avoir réalisé une formation pratique supervisée (Ressources humaines et Développement des compétences Canada, 2017b). Au Canada, les programmes d'études en en pratique sage-femme mènent à un baccalauréat et ne prévoient aucun critère d'admissibilité. Sept de ces programmes sont actuellement reconnus; ils sont offerts par des universités en Colombie-Britannique, en Ontario, au Québec, au Manitoba et en Alberta (Association canadienne des sages-femmes, 2015a). En 2014, on comptait au pays plus de 1200 sages-femmes inscrites auprès d'une association provinciale (Association canadienne des sages-femmes, 2014). La plupart d'entre elles pratiquent en Ontario, en Colombie-Britannique et au Québec.

\section{Le coût des MAC au Canada}

En analysant les coûts engagés par les patients pour utiliser des MAC, on constateque les services des sages-femmes et des chiropraticiens ont été au moins partiellement intégrés à ceux de la biomédecine conventionnelle, tandis que la naturopathie et l'homéopathie demeurent en marge. En effet, tous les territoires qui réglementent la pratique sage-femme l'ont incluse dans leur système public de soins de santé; autrement dit, les femmes enceintes peuvent obtenir gratuitement les services d'une sage-femme. Signalons également que la plupart des régimes d'assurance de soins de santé remboursent les services de chiropratique, même s'ils ne sont pas couverts par les programmes publics (ACC, 2015a). Après la première consultation auprès d'un chiropraticien, le coût moyen des consultations suivantes varie entre 40 et 60 \$ (Alberta College and Association of Chiropractors, 2015). À l'opposé, aucun régime provincial d'assurance maladie ne couvre les services de naturopathes et d'homéopathes (ACDN, 2017d; Canadian Society of Homeopaths, 2015). Selon l'ACDN, une consultation auprès d'un docteur en naturopathie coûte entre 35 et 250 , en fonction de sa durée; le barème des frais de l'ACDN est établi sur la base d'une rémunération horaire variant entre 125 et $180 \$$ (ACDN, 2017d). La première consultation auprès d'un homéopathe coûte 150 \$ ou plus, selon l'expérience du praticien (Canadian Society of Homeopaths, 2015). On notera cependant que si les services homéopathiques sont offerts par un médecin (ce qui arrive parfois au Canada), leur coût est absorbé par le régime public. La plupart des médicaments homéopathiques sont accessibles sans ordonnance en pharmacie et leur coût est relativement faible. 


\section{$M A C$, vaccination et réticence à la vaccination ${ }^{3}$}

On ne peut douter de l'influence qu'exercent les recommandations des professionnels de la santé issus de la biomédecine sur l'acceptabilité de la vaccination (Williams, 2014; Yaqub et coll., 2014). On comprend toutefois moins bien comment les praticiens des MAC peuvent influencer la décision vaccinale de leurs patients (McMurtry et coll., 2015). Comme les Canadiens sollicitent de plus en plus les praticiens des MAC pour obtenir un traitement médical et comme ces praticiens présentent souvent des attitudes plus négatives à l'égard de la vaccination, il devient crucial de bien connaître leurs opinions à ce sujet (Bean et Catania, 2013). C'est ainsi seulement que l'on pourra mieux comprendrel'effet combiné des sous-cultures réticentes à la vaccination.

\section{Attitudes et pratiques des utilisateurs des MAC en matière de vaccination}

Dans littérature internationale, l'association entre l'utilisation des MAC et le refus de la vaccination chez les adultes et leurs enfants a fréquemment été observé (Cassell et coll., 2006; Gaudino et Robison, 2012; Zuzak et coll., 2008; Downey et coll., 2010; Jones, Sciamanna et Lehman, 2010; Smith et coll., 2011). D'autres étudess ont cependant indiqué que cette association pourrait résulter du fait que les familles réticentes à la vaccination choisissent d'être suivies par des praticiens des MAC plutôt que du fait que ces praticiens recommandent d'éviter la vaccination (Kriwy, 2012; Salmon et coll., 2005).

Plusieurs études réalisées au Canada ont révélé que le recours aux MAC est associé à une attitude négative vis-à-vis de la vaccination, comme l'ont indiqué Guay, Dubé et Laberge (se reporter au chapitre 6 du présent ouvrage). Par exemple, dans l'une de ces études réalisée en 2011, 16 p. cent des parents étaient d'accord pour dire que l'utilisation de pratiques médicales alternatives pourrait éliminer la nécessité de la vaccination (Les Associés de recherche EKOS inc., 2011). Deux études canadiennes portant sur les facteurs déterminants de la couverture vaccinale ont aussi démontré une association entre l'accouchement avec l'aide d'une sagefemme et le statut vaccinal incomplet de l'enfant (Zhang et coll., 2008; Guay et coll., 2009). Dans l'une de ces études, la probabilité qu'un enfant ait un statut vaccinal incomplet à l'âge de 24 mois était sept fois plus grande si l'accouchement avait été supervisé par une sagefemme plutôt que par un médecin (Guay et coll., 2009).

Dans une enquête par questionnaire, réalisée en 2010 auprès de 95 parents d'enfants âgés de seize ans ou moins ayant consulté une clinique ontarienne de naturopathie, à peine 50 p. cent des répondants ont affirmé que leurs enfants avaient reçu tous les vaccins recommandés. Différentes études ont illustré que le fait de percevoir une pression pour accepter la vaccination de la part du médecin de famille ou du pédiatre et, d'autre part, le fait de considerer le naturopathe comme la source d'information sur la vaccination la plus digne

3. Cette section s'inspire d'une revue de la littérature faite au moyen de la base de données PubMed, selon les données disponibles le 19 septembre 2012 et mises à jour le 16 mars 2015. Nous avons utilisé différents mots-clés pour cerner les études portant sur la connaissance de la vaccination, les attitudes à cet égard et les pratiques des utilisateurs et des praticiens des MAC; nous avons accordé une attention particulière aux études canadiennes. 
de confiance étaient associés à la vaccination incomplète ou à la non vaccination des enfants (Busse, Walji et Wilson, 2011; Zhang et coll., 2008).

En 2002, des chercheurs ont examiné le dossier médical de 482 patients âgés de moins de dix-huit ans (âge moyen de 6,5 ans) de la clinique de naturopathie du Canadian College of Naturopathic Medicine de Toronto (Wilson, Busse et coll., 2005). De ces dossiers, 316 contenaient de l'information sur la vaccination et, parmi ceux-ci, 86,7 p. cent indiquaient que l'enfant avait reçu tous les vaccins recommandés, 4,4 p. cent notaient une vaccination partielle et 8,9 p. cent signalaient une absence totale de vaccins. Cette étude a illustré que le jeune âge de l'enfant, le recours plus fréquent aux MAC et la visite de cliniques de MAC pour obtenir de l'information sur la vaccination étaient associés à la vaccination incomplète ou à la non-vaccination des enfants (Wilson, Busse et coll., 2005).

Dans une étude qualitative longitudinale réalisée auprès de 56 mères québécoises, nous avons établi que de nombreuses mères réticentes à la vaccination, qui avaient décidé de refuser ou de retarder la vaccination, recouraient plus ou moins fréquemment aux MAC. Ces mères avaient l'impression que l'information transmise par les autorités de santé publique et le gouvernement était «trop en faveur de la vaccination », tandis que la critique provenant des groupes « anti-vaccination » était «trop opposée à la vaccination ». À la recherche d'une information plus «équilibrée », certaines d'entre elles se sont tournées vers les praticiens des MAC. Elles considéraient leurs recommandations comme étant plus crédibles que celles des experts en santé publique, des infirmières ou des médecins, car elles jugeaient que ces praticiens n'avaient rien à perdre, ni à gagner en matière de vaccination (Dubé et coll., 2016), comme le dit cette mère indécise, âgée de 31 ans :

Le chiropraticien n'en tire aucun avantage. Je ne vois pas quel profit les gens auraient à promouvoir des idées allant à l'encontre de la vaccination, sauf s'il s'agit de leur propre opinion et de leurs propres convictions; il n'y a aucun bénéfice économique pour eux $(746)^{4}$.

\section{MAC et vaccination : la position des praticiens}

Dans certaines études canadiennes, on a directement interrogé des praticiens des MAC au sujet de leurs connaissances de la vaccination, ainsi que de leurs attitudes et de leur pratique à cet égard. La plupart de ces études avaient pour sujets des chiropraticiens et leurs résultats laissent généralement entrevoir que bien des praticiens des MAC montrent une hésitantion vis-à-vis la vaccination. Dans les pages qui suivent, nous présenterons brièvement les positions officielles qu'ont prises, le cas échéant, les associations canadiennes de praticiens des MAC, ainsi que les conclusions d'études pertinentes réalisées auprès de sages-femmes, de naturopathes, d'homéopathes et de chiropraticiens canadiens.

4. Nous avons présenté des témoignages de mères ayant été interviewées dans le cadre d'une étude qualitative longitudinale réalisée au Québec en 2011-2012. Cette étude a été faite avec l'aide financière des Instituts de recherche en santé du Canada (MOP-115012). On peut trouver plus de renseignements sur la méthodologie et les résultats dans Dubé et coll. (2016). 


\section{Naturopathie et vaccination}

Dans ma propre vision de la vie, je pense que nous en faisons trop ... tandis que la nature fait bien les choses ... je n'ai pas fait vacciner ma fille et cela ne me cause aucun problème, parce que j'ai une perspective personnelle au sujet de la santé naturelle. À mon avis, plus nous intervenons, moins bons seront les résultats. J'estime que ma fille possède ses propres anticorps et qu'il n'y a rien à craindre.

- Mère de 28 ans, réticente à la vaccination et ayant refusé tous les vaccins pour son enfant

Peu d'information sur la vaccination était disponible sur le site Web de l'ACDN et depuis, cette information a été retirée du site. Par exemple, en 2012, on y trouvait la description suivante de la position de l'association sur le vaccin antigrippal :

La vaccination empêche le corps de réagir naturellement à des pathogènes externes tels que les virus et les bactéries. Les personnes dont le système immunitaire est fort et sain se protégeront elles-mêmes des effets négatifs de la grippe, acquerront une immunité permanente et risqueront moins d'être affectées par les effets indésirables encore inconnus d'une exposition à long terme aux vaccins. (ACDN, 2004)

Avant d'expliquer comment prévenir la grippe à l'aide d'une bonne nutrition et de mesures d'hygiène, on concluait sur ces mots:

Le vaccin antigrippal a été mis au point par le système de soins de santé allopathique afin d'atténuer le risque de contracter le virus de la grippe. La meilleure manière d'éviter la grippe et ses complications réside toutefois dans la prévention. Celle-ci doit reposer principalement sur l'acquisition de saines habitudes quotidiennes qui optimisent le système immunitaire et la santé globale. Un solide système immunitaire représente la stratégie la plus efficace pour contrer tous les virus, y compris celui de la grippe (ACDN 2004)

Quatre études réalisées au Canada auprès d'étudiants en naturopathie ont démontré que la plupart d'entre euxétaient hésitants, en matière de vaccination (Wilson et coll., 2005a; Wilson et coll., 2004; Busse, Wilson et Campbell, 2008; McMurtry et coll., 2015). Dans la première étude, on a remis aux 525 étudiants en $4^{\text {ème }}$ année du programme de naturopathie du Canadian College of Naturopathic Medicine un questionnaire portant sur leurs connaissances de la vaccination et leurs attitudes à cet égard (Wilson et coll., 2004). Parmi les 312 répondants, à peine 12,8 p. cent ont déclaré avoir l'intention de recommander à leurs patients de recevoir tous les vaccins recommandés; 74,4 p. cent en recommanderaient quelques-uns seulement. L'étude concluait également que l'intention de recommander tous les vaccins et la confiance envers les autorités de santé publique et la médecine conventionnelle diminuaient en fonction du nombre d'années de scolarité terminées (Wilson et coll., 2004). Une sous-étude s'adressant aux étudiants de dernière année seulement $(n=97)$ a donné des résultats similaires même après des interventions particulières de promotion de la vaccination (Wilson et coll., 2005b).

Enfin, on a formé onze groupes de discussion avec 62 étudiants en médecine, en chiropratique et en naturopathie (Busse, Wilson et Campbell, 2008; McMurtry et coll., 2015). L'analyse préliminaire des résultats des groupes de discussion réunissant des étudiants en chiropratique et en naturopathie a montré que bon nombre d'entre eux étaient préoccupés au sujet des effets à long terme des vaccins, exprimaient des doutes à l'effet que le système

5. Cet énoncé de position ne figure plus sur le site Web de l'ACDN depuis mars 2015. 
immunitaire de l'enfant pouvait être surchargé par de multiples vaccins, ne trouvaient pas crédibles les données sur les risques et les avantages de la vaccination provenant des fabricants de vaccins, exprimaient des doutes au sujet de certains ingrédients entrant dans la fabrication et croyaient que différentes approches holistiques (comme l'homéopathie) pouvaient donner une protection similaire ou meilleure que celle de la vaccination (Busse, Wilson et Campbell, 2008). Bramadat, O'Doherty et Brunk, aux chapitres 1, 2 et 3 du présent ouvrage, ont montré que ces inquiétudes reflétaient une angoisse plus profonde chez les individus hésitants. L'analyse finale des résultats des groupes de discussion illustrait le développement des perspectives sur la vaccination chez les étudiants en chiropratique et en naturopathie et permettait de les comparer à celles des étudiants en médecine (McMurtry et coll., 2015). Les conclusions démontrent que les étudiants ont entrepris leur formation professionnelle en ayant l'esprit ouvert au sujet de la vaccination et que leurs études combinées à la socialisation informelle ont eu d'importantes répercussions sur leurs convictions; la plupart des étudiants, dans les premières années de leur formation, acceptaient en effet sans contestation l'opinion exprimée par les professionnels de la biomédecine dont ils respectaient la réputation. En conclusion, les étudiants convenaient, en majorité, de l'importance d'informer les patients et de la valeur des données probantes de la médecine scientifique entourant la vaccination. Les chercheurs ont pourtant constaté de grandes différences dans l'importance donnée à d'autres éléments : tandis que les étudiants en médecine mettaient de l'avant les effets à plus grande échelle de la vaccination sur la santé publique, ceux qui étudiaient la chiropratique ou la naturopathie "plaidaient en faveur de bons choix en matière de mode de vie, de la liberté individuelle et de facteurs 'holistiques' » (McMurtry et coll., 2015, 1299).

\section{Homéopathie et vaccination}

J'ai consulté une homéopathe au sujet des coliques de mon enfant et nous avons parlé de la vaccination; c'est pourquoi j'ai choisi de reporter des vaccins.

[Intervieweur] Que vous a conseillé l'homéopathe au sujet de la vaccination?

Elle m'a donné trois possibilités : reporter la vaccination, administrer à mon enfant des vaccins homéopathiques pendant cinq ans ou lui faire donner certains vaccins réguliers et employer des vaccins homéopathiques pour les autres. J'avais donc quelques possibilités ... elle ne donne pas de vaccins ... aucun de ses enfants n'est vacciné, vous savez ... elle n'a rien contre les vaccins, mais ...

- Mère réticente, âgée de 35 ans

J'ai consulté une homéopathe pour obtenir une trousse de préparation aux premiers vaccins pour ma fille. C'est un produit homéopathique qui stimule le système immunitaire avant l'administration de la charge virale des vaccins.

[Intervieweur] Ah! II y a donc des produits homéopathiques de préparation à la vaccination?

Oui, ça se voit fréquemment... Cette homéopathe était absolument opposée à la vaccination. Son discours était très clair... elle avait beaucoup d'arguments contre la vaccination. 
Malgré le fait que le créateur de l'homéopathie était un fervent partisan de la vaccination (Boyer et coll., 2006), de nombreuses études, réalisées dans différents pays, ont démontré que les homéopathes conseillent à leurs patients de l'éviter ${ }^{6}$. Aucune étude n'a cependant été réalisée chez les homéopathes canadiens.

Les homéopathes peuvent recommander des vaccins homéopathiques, qu'ils appellent « nosodes » et qui sont des agents infectieux très dilués administrés par la bouche. Les nosodes sont constitués à partir de matières biologiques comme du pus, des tissus contaminés, du sang, de la salive ou des bacilles provenant d'excréments (Pray, 1996). Certains homéopathes les recommandent comme substituts aux vaccins biomédicaux, afin de prévenir les maladies contagieuses pouvant affecter les enfants ou les voyageurs (Birch, 2015). II est intéressant de noter que certains de ces produits, comme l'Influenzinum qui est utilisé pour prévenir la grippe, sont homologués en vertu du Règlement sur les produits de santé naturels de Santé Canada. Cette démarche d'homologation a subi de cinglantes critiques des experts, car elle confère une image d'efficacité à des produits qui, selon les données scientifiques, sont incapables de traiter ou de prévenir quelque condition médicale que ce soit (Picard, 2015; Weeks, 2015a, 2015b). En juin 2013, Santé Canada a ordonné qu'on ajoute une mise en garde à l'étiquetage standardisé des nosodes, dans les termes suivants : "Ce produit n'est pas un vaccin ni une alternative à la vaccination. L'efficacité de ce produit n'a pas été prouvée pour la prévention d'aucune infection. Santé Canada ne recommande pas son utilisation chez les enfants et conseille que votre enfant reçoive tous les vaccins courants. » (Santé Canada, 2015) Malgré l'adoption de cette nouvelle norme, les praticiens peuvent tout de même les prescrire comme substituts à la vaccination, car les droits, en matière d'ordonnance, ne relèvent pas des pouvoirs d'exécution de la loi de ce ministère.

Les défenseurs de l'homéopathie affirment que les vaccins homéopathiques sont « naturels » et «non toxiques » et qu'ils n'ont pas d'effets indésirables. Ils incitent le corps à produire une forte réaction immunitaire, afin de se protéger contre la maladie.

\section{Quels succès remportent les nosodes pendant les é pi d ém i es?}

Lors de flambées épidémiques survenues au cours des derniers siècles, on a eu recours avec succès à l'homéopathie dans différents pays. À Cuba, par exemple, on a récemment réussi à prévenir la leptospirose, une maladie tropicale, en administrant des vaccins homéopathiques (nosodes) à des millions de personnes. Au Brésil, on a utilisé le même moyen pour prévenir la méningite chez des milliers de citoyens (McLeod, 2014).

On notera cependant que le manque de renseignements méthodologiques sur les études d'où sont tirées ces données restreint la possibilité de réfuter ou de confirmer ces allégations sur une base scientifique. Par ailleurs, les références qui les appuient ne proviennent pas de publications revues par un comité de lecture; il s'agit plutôt de sites Web faisant la promotion de vaccin homéopathique, d'articles de revues spécialisées en homéopathie et de reportages publiés au début du XXe siècle, qui ne sont pas facilement accessibles.

6. Se reporter à Ernst (2001) pour une vue d'ensemble de ces études. 
Malgré l'apparente rigueur scientifique conférée à ces renseignements, les essais cliniques, en homéopathie, ne se comparent pas favorablement à ceux réalisés en biomédecine, du point de vue de la qualité (Société canadienne de pédiatrie, 2005). Comme le laisse entendre Bramadat au chapitre 1 du présent ouvrage, la plupart des hypothèses de la théorie homéopathique (par exemple la notion voulant que l'absence d'un ingrédient actif dans un médicament lui donne plus de puissance que sa présence) ressemblent à des convictions de fidèles, car elles contredisent des preuves reconnues émanant de la recherche et de la pratique en microbiologie. Ajoutons à cela que la biomédecine n'a pas produit jusqu'ici de données scientifiques fiables démontrant l'efficacité des produits homéopathiques pour la prévention des maladies contagieuses (NHMRC, 2015a). En outre, certains articles de revues scientifiques ont rapporté que des personnes auraient contracté ces maladies après une « vaccination » homéopathique (Ernst, 2001). En fait, la possibilité que l'homéopathie puisse un jour accéder à la légitimité scientifique a été réduite à néant par une solide méta-étude publiée dans The Lancet en 2005 (Shang et coll., 2005). Le même résultat a été obtenu lors de l'examen, par l'Australian National Health and Medical Research Council (NHMRC), des données portant sur l'efficacité de l'homéopathie dans le traitement de toute condition clinique chez l'homme. Suite à l'évaluation des données probantes entourant l'efficacité de l'homéopathie, l'organismeconcluait ainsi : «... nous n'avons trouvé aucun état de santé pour lequel il existe des données fiables prouvant l'efficacité de l'homéopathie. » (NHMRC, 2015a, 6) II déclarait en plus que «l'homéopathie ne devrait jamais être utilisée pour traiter des maladies chroniques, graves ou susceptibles de le devenir. Les gens qui choisissent l'homéopathie risquent de nuire à leur santé s'ils rejettent ou reportent des traitements dont l'innocuité et l'efficacité ont été prouvées. » (NHMRC, 2015b)

\section{Chiropratique et vaccination}

J'ai refusé que mes enfants soient vaccinés et je suis sûre d'avoir pris la bonne décision.

[Intervieweur] Parlez-vous des vaccins avec vos patients?

Oui, comme je le fais pour d'autres sujets comme l'allaitement. Je leur donne de bonnes études à parcourir et des renseignements qui ont influencé ma propre décision au sujet de la vaccination. Je ne recommande pas de refuser la vaccination, car je crois que cette décision est personnelle. Je leur ai transmis des renseignements pour qu'ils puissent prendre une décision éclairée.

- Chiropraticienne mère de trois enfants non vaccinés

La documentation rattachée à la chiropratique expose de nombreux arguments contre la vaccination. Celle-ci est souvent décrite comme dangereuse et inefficace (Ernst, 2001, 2011). Le site de l'International Chiropractic Pediatric Association (http://icpa4kids.org/), notamment, présente en grande partie les risques de la vaccination et contient de nombreux hyperliens menant à d'autres sites qui lui sont opposés. En revanche, l'Association chiropratique canadienne (ACC) ne prend aucune position officielle contestant l'immunisation. Elle déclare plutôt ce qui suit (ACC, 2015d) : 
La vaccination est une procédure bien connue et largement recommandée par les autorités de santé publique; l'Association chiropratique canadienne appuie les stratégies de promotion de la santé publique et de prévention qui favorisent la santé et le bien-être sur le plan physique et mental. L'ACC convient que la vaccination est une procédure de prévention financièrement rentable et cliniquement efficace pour certaines maladies causées par des virus et des microbes, comme l'ont démontré les milieux scientifiques. La responsabilité publique à l'égard de la vaccination et de l'immunisation ne relève pas de la pratique chiropratique et ne constitue pas une question d'importance pour les chiropraticiens. Les programmes de santé publique et la documentation sont des sources appropriées d'information des patients au sujet de la vaccination et de l'immunisation.

Malgré la position favorable exprimée par l'ACC, la vaccination fait toujours l'objet d'une controverse parmi les chiropraticiens et un petit nombre d'entre euxy sont fortement opposés et l'expriment ouvertement (Busse et coll., 2002; Gleberzon et coll., 2013). Campbell, Busse et Injeyan (2000), à l'instar de Busse, Morgan et Campbell (2005), ont cerné plusieurs arguments anti-vaccination qu'utilisent fréquemment les chiropraticiens : les vaccins sont inefficaces, puisque des maladies contagieuses apparaissent même dans les collectivités vaccinées et que leur diminution est attribuable à une amélioration des conditions de vie et de l'hygiène; la vaccination n'est pas nécessaire et peut même être dangereuse; on voit même dans les milieux médicaux une mésentente au sujet de l'utilité des vaccins, voire une contestation de la vaccination. Ce genre de discours se retrouve dans des articles de revues de chiropratique (non soumis à un comité de lecture), sur les sites Web de chiropraticiens et dans la publicité payante (Busse, Morgan et Campbell, 2005).

Les études réalisées auprès de chiropraticiens canadiens montrent également que les professionnels réticents ou opposés à la vaccination sont nombreux. En l'an 2000, une enquête par questionnaire a été menée auprès de 467 des 621 étudiants du Canadian Memorial Chiropractic College (CMCC) (Busse et coll., 2002). Selon cette étude, certains étudiants de première année montraient une attitude négative à l'égard de la vaccination et la proportion qu'ils représentaient augmentait avec les années de formation. Chez les étudiants de quatrième année, par exemple, on convenait dans des proportions de 24 et de 17 p. cent « qu'il n'y avait guère de preuves scientifiques démontrant que l'immunisation prévient les maladies contagieuses » et «que les vaccins n'ont pas considérablement modifié l'incidence des principales maladies contagieuses », alors que les proportions étaient de 2,7 et de 6,2 p. cent pour les étudiants de première année. Bien que les méthodes transversales ne permettent pas de tirer des conclusions définitives au sujet des effets de la formation sur le développement d'attitudes négatives chez les étudiants en chiropratique, ces résultats laissent entrevoir une tendance inquiétante (Busse et coll., 2002) qui s'apparente à celle qui s'est dégagée chez les étudiants en naturopathie (Wilson et coll., 2004). En 2011, une étude similaire a été réalisée auprès de 368 des 760 étudiants du CMCC et les résultats ont été fort différents (Lameris et coll., 2013). Ils ont montré qu'entre 84 et 90 p. cent des étudiants inscrits dans les différentes années exprimaient des attitudes positives vis-à-vis de la vaccination; Busse et ses collègues, en 2002, avaient fixé à 73 p. cent le plus haut résultat positif. De surcroît, l'étude de Busse indiquait que les étudiants de quatrième année montraient des attitudes plus négatives que ceux de première année, mais l'étude de Lameris a souligné que les étudiants de chacune des quatre années affichaient une attitude globalement favorable à la vaccination. Il est impossible, à partir de ces deux enquêtes transversales, de conclure qu'il se forme une tendance en faveur d'attitudes plus favorables à l'égard de la vaccination chez les étudiants en chiropratique. Par ailleurs, alors que la plupart des étudiants ont répondu au 
questionnaire de Busse, moins de la moitié l'a fait pour le questionnaire de Lameris. Ainsi, il est possible qu'un biais dans les réponses favorisant les étudiants ayant une attitude plus positive envers la vaccination existe pour l'étude de Lameris (Gleberzon et coll., 2013).

Un autre questionnaire a été envoyé par la poste aux 643 chiropraticiens albertains; 503 y ont répondu. Ce sondage comportant cinquante-cinq questions avait pour but de cerner les pratiques et les attitudes des chiropraticiens à l'égard de la vaccination (Russell et coll., 2004; Page et coll., 2006; Medd et Russell, 2009; Russell, Verhoef et Injeyan, 2005). Les résultats montrent que $45 \mathrm{p}$. cent des chiropraticiens discutent régulièrement de la vaccination avec leurs patients (10 p. cent le font une fois par semaine et $35 \mathrm{p}$. cent chaque mois). Ce groupe avait en outre une attitude plus négative envers la vaccination que ceux qui en discutaient moins souvent avec leurs patients. Parmi les pratiques les plus courantes des chiropraticiens en matière de vaccination figuraient un rappel indiquant que celle-ci demeure un choix personnel (70 p. cent des chiropraticiens ont dit avoir fait ce rappel dans les six mois précédant le sondage) et la réponse aux questions ou la remise d'information sur les risques associés à la vaccination (proportion de 67,8 p. cent). Enfin, malgré que cela soit contraire à la position officielle de l'ACC, 27,2 p. cent des répondants ont déclaré avoir recommandé à leurs patients d'éviter la vaccination, pour eux-mêmes ou pour leurs enfants. Une proportion similaire de répondants a cependant affirmé recommander la vaccination (Russell et coll., 2004). Un peu plus de 60 p. cent des chiropraticiens ont exprimé une certaine ouverture à l'idée de collaborer avec les médecins et les infirmières de santé publique, dans le but principal d'obtenir d'eux de l'information sur la vaccination.

Des entrevues ont également été réalisées auprès de quatorze répondants afin de mieux connaître leur approche en matière de vaccination auprès de leurs patients (Page et coll., 2006). Les résultats de cette étude montrent que la discussion sur la vaccination était généralement entamée par le patient, parfois à la suite d'une communication indirecte (brochures ou articles accessibles en salle d'attente). Un seul des chiropraticiens a recommandé à ses patients de se faire vacciner; les autres ont dit avoir fourni de l'information, pour qu'ils puissent prendre une décision éclairée. Certains chiropraticiens s'étaient expressément donné pour tâche d'expliquer aux patients l'« autre côté » de la vaccination (Page et coll., 2006).

Des analyses supplémentaires ont porté sur les liens entre le statut vaccinal des chiropraticiens, celui de leurs enfants et leurs recommandations aux patients (Medd et Russell, 2009). Seuls les répondants ayant au moins un enfant ont été inclus ( $n=325)$. Parmi ceux qui n'avaient qu'un enfant ( $n=63), 16$ p. cent ont déclaré que l'enfant avait reçu tous les vaccins recommandés, 57 p. cent qu'il n'avait reçu que certains vaccins et 27 p. cent qu'il n'avait reçu aucun vaccin. Chez les répondants ayant plusieurs enfants, les réponses sur le statut vaccinal de l'aîné étaient réparties assez également entre les trois catégories. Il est intéressant de noter qu'environ le quart des chiropraticiens dont le premier enfant avait reçu tous les vaccins ont pris d'autres décisions pour le ou les enfants suivants. Seulement 22 p. cent des chiropraticiens ont indiqué qu'ils recommandaient à leurs patients de faire vacciner leurs enfants, une proportion beaucoup plus faible que celle observée dans des études menées auprès de médecins et de pédiatres canadiens, dont la quasi-totalité recommandait la vaccination (Dubé et coll., 2012).

En 2010, une autre enquête auprès d'un échantillon de 740 chiropraticiens canadiens a été réalisée; le taux de réponse fut de 68 p. cent et le questionnaire portait notamment sur les attitudes et les comportements vis-à-vis de la vaccination (Puhl et coll., 2014; McGregor 
et coll., 2014). Dans ce cas-ci, les résultats montrent une proportion considérablement plus faible de chiropraticiens recommandant aux parents d'éviter de faire vacciner leurs enfants, comparativement à l'étude albertaine (7 p. cent contre 27 p. cent) (Russell et coll., 2004). Les chiropraticiens répondaient le plus souvent qu'ils conseillaient aux parents de rencontrer un médecin ou une infirmière pour se renseigner sur les vaccins pédiatriques (39 p. cent) ou donnaient les avantages et les inconvénients de la vaccination des enfants (38p. cent). L'étude permet également de supposer que la plupart des chiropraticiens canadiens convenaient «qu'il y avait de bonnes données probantes pour appuyer la théorie de la vaccination » et «que la vaccination avait amélioré la santé de la population » (Puhl et coll., 2014, 715). Elle montre aussi que les attitudes à l'égard de la vaccination sont fortement liées aux concepts idéologiques sous-tendant les établissements où les répondants ont reçu leur formation en chiropratique, les attitudes négatives se retrouvant probablement plus souvent chez les diplômés des établissements américains conservateurs (Puhl et coll., 2014; McGregor et coll., 2014).

\section{Pratique sage-femme et vaccination}

Elle [la sage-femme] m'a tout simplement laissée prendre ma propre décision. Elle m'a dit qu'il n'y avait pas de problème si je refusais la vaccination; tout ça s'est fait très vite, en à peine trente secondes.

- Mère opposée à la vaccination, âgée de 33 ans

Je n'aime pas beaucoup les gens qui pensent en blanc ou en noir. C'est pourquoi j'ai apprécié l'approche de la sage-femme. Même si je savais qu'elle était opposée à la vaccination et que ses enfants n'étaient pas vaccinés, elle m'a donné les renseignements demandés, et sans partisanerie. Je me méfie toujours de l'information qui est trop en faveur de la vaccination ou trop contre. À mon avis, la couleur de la vie est plus grise que noire ou blanche.

- Mère de 28 ans, réticente et ayant refusé tous les vaccins pour son enfant

Examinons maintenant la relation entre la pratique sage-femme et la vaccination. Nous avons, à cette fin, évalué deux études canadiennes portant sur ce sujet. En 2002, une recherche ontarienne (Lee et coll., 2005) s'est penchée sur les convictions et les pratiques des sages-femmes et des étudiantes dans le domaine. II en est ressorti que 27 p. cent des sagesfemmes ontariennes avaient reçu le vaccin contre la grippe, contre 60 p. cent de l'ensemble des travailleurs de la santé durant la même saison. Parmi les sages-femmes en exercice et les étudiantes, 56 p. cent ont déclaré être en faveur du vaccin antigrippal, tandis que 34 p. cent demeuraient neutres. Par ailleurs, 24 p. cent des sages-femmes ont indiqué qu'elles recommandaient la vaccination à leurs clients, contre 64 p. cent qui ne se prononçaient pas.

On a fait une analyse qualitative auprès de sages-femmes québécoises afin de décrire leurs pratiques, attitudes et convictions en matière de vaccination (Dubé, Vivion, Valderrama et coll., 2013; Dubé, Vivion, Sauvageau et coll., 2013). Vingt-cinq entrevues ont été faites en 2010, dont 17 avec des sages-femmes en exercice et 8 avec des étudiantes. L'étude a mis en lumière l'importance de la philosophie adoptée par la sage-femme, car elle guide toute sa pratique. Le choix éclairé figure parmi les principes clés de la pratique sage-femme moderne, sur lequel se 
basent les sages-femmes dans leur approche vis-à-vis les questions de promotion de la santé, dont la vaccination. Les infirmières et les médecins québécois sont tenus de recommander la vaccination à leurs patients (ACPM, 2008; Durand, Allard et Thibault, 2007); les sagesfemmes faisant l'objet de l'étude cherchaient plutôt à fournir de l'information et présentaient les avantages et les inconvénients de la vaccination aux parents. La plupart d'entre elles ont ainsi déclaré qu'elles leur remettaient de la documentation gouvernementale, dont le guide Mieux vivre avec notre enfant de la grossesse à deux ans (https://www.inspq.qc.ca/ publications/2052) et différents documents, y compris sur la naturopathie (Arsenault, 2004, 2009). Les sages-femmes reconnaissaient généralement les avantages de la vaccination, notamment son efficacité dans la prévention des maladies et le fait qu'elle soit gratuite; elles avaient cependant plusieurs arguments à faire valoir à son encontre, particulièrement en ce qui a trait au calendrier, qui selon certaines commence trop tôt dans la vie du nourrisson, et à la combinaison des vaccins, qui nuit au libre choix des parents. Soulignons que toutes les sages-femmes interviewées ont déclaré que leur opinion personnelle sur la vaccination n'a en aucun cas influencé la discussion avec les patients. Un certain nombre des répondantes a mentionné qu'il était difficile de trouver une information nuancée sur la vaccination. Un second volet de l'étude, quantitatif celui-là, a été mené auprès de sages-femmes en exercice et d'étudiantes en pratique sage-femme. Malgré un faible taux de réponse (46 répondantes sur 131) et la difficulté qu'ont éprouvée certaines sages-femmes à répondre au questionnaire, l'étude a confirmé que la vaccination est sujette à controverse dans ce milieu et qu'un petit nombre de répondantes a mentionné la nécessité d'une formation à ce sujet (Vivion et coll., 2011).

\section{Conclusions}

D'une façon ou d'une autre, si mon homéopathe me dit que ma fille va attraper le cancer, puisqu'il semble que la vaccination donne le cancer, ou si le gouvernement me dit qu'il y a encore des gens qui meurent faute d'avoir été vaccinés... tout ça, pour moi, c'est de la partisanerie; je n'aime pas ça et c'est pourquoi je n'ai pas encore pris de décision. Cette information est bien difficile à accepter.

- Mère réticente, âgée de 28 ans

Les données présentées dans ce chapitre indiquent que de nombreuses MAC se sont engagées dans un processus de professionnalisation (Fadlon, 2005) afin " d'être moins marginaux dans le milieu actuel des soins de santé » (Kelner et coll., 2006, 2617). Kelner et ses collègues (2006) expriment l'avis que ce processus est fondé sur quatre grandes stratégies: améliorer les programmes d'études, rehausser les normes de pratique, accroître le nombre d'études soumises à un comité de lecture et encourager la cohésion entre les praticiens. Comme nous l'avons mentionné précédemment, les chiropraticiens et les sages-femmes ont réussi à s'intégrer dans le système de soins de santé « officiel » du Canada et ils acquièrent une influence correspondant à leur présence croissante. Une analyse récente du contenu des sites Web de grands collèges, associations et cliniques de chiropratique montre que « le passage de la chiropratique de la périphérie au rang des services réguliers » est à l'origine d'un discours où elle est présentée simultanément comme « une pratique fondée sur des données probantes et respectant les mêmes conventions de la science traditionnelle » et « une autre option qui adopte une approche naturelle » (Shelley, Clark et Caulfield, 2015, 20). 
Malgré que les naturopathes et les homéopathes n'aient pas encore atteint le degré d'intégration dans la biomédecine que l'on constate chez les chiropraticiens et les sagesfemmes, la réglementation de la pratique homéopathique adoptée par l'Ontario et I'homologation des produits homéopathiques par Santé Canada donnent certainement l'impression que le gouvernement les approuve. Ces décisions ont reçu un mauvais accueil chez les défenseurs de la vaccination (MacDonald et coll., 2011; Ernst, 2015b). Ceux-ci, invoquant le manque de preuves de l'efficacité de ces produits (Shang et coll., 2005; NHMRC, 2015a), allèguent que les processus d'homologation et de réglementation auront pour effet de transmettre à la population des messages ambivalents, pouvant signifier que l'homéopathie soit aussi efficace que les traitements biomédicaux comme les vaccins.

Nous avons aussi démontré qu'une proportion considérable de professionnels des MAC, au Canada, sont réticents ou opposés à la vaccination. Certains professionnels de la biomédecine - les infirmières, en particulier - ressemblent certes à leurs homologues praticiens des MAC à cet égard, notamment en ce qui concerne les vaccins antigrippaux (Dubé, Defay et coll., 2011; Dionne et coll., 2001), mais les médecins de famille et surtout les pédiatres sont pour la plupart des défenseurs convaincus de la vaccination (Daley et coll., 2006; Posfay-Barbe et coll., 2005; Kempe et coll., 2009; Dubé, Gilca et coll., 2011). Dans une perspective biomédicale, la position des professionnels des MAC à ce sujet peut être inquiétante, car il a été démontré que la vaccination figure parmi les méthodes efficaces de prévenir les décès et les complications de maladies évitables par un vaccin. La réticence et l'opposition à la vaccination risquent parfois de présenter un problème pour les professionnels de la biomédecine qui ont tendance à considérer les craintes et les inquiétudes de leurs patients comme irrationnelles, inspirées par la superstition, mal informées ou stimulées par des mouvements anti-vaccination (FlanaganKlygis, Sharp, et Frader, 2005; Opel et coll., 2012; Leib, Liberatos et Edwards, 2011). Quelques études ont souligné que les parents trouvent parfois difficile de discuter ouvertement au sujet de la vaccination avec leur médecin et disent se sentir aliénés lorsqu'on discute de vaccins (Opel et coll., 2012). À l'opposé, ces discussions tenues auprès de praticiens de la médecine alternative tels que les naturopathes semblaient davantage correspondre à ce que ces parents considéraient comme une consultation idéale au sujet de leurs enfants, par rapport aux consultations avec un médecin (Busse, Walji et Wilson, 2011; Dubé, Vivion, Sauvageau et coll., 2013).

Nous avons indiqué dans ce chapitre ce que soulignent d'autres études (Bean et Catania, 2013; McMurtry et coll., 2015), à savoir que la position des professionnels des MAC et de la biomédecine se développe en partie sous l'effet des influences normatives socioprofessionnelles ayant des répercussions sur les comportements lors de la vaccination, les convictions au sujet de la santé et de la prévention et la confiance que l'on a dans les différentes sources d'information sur les vaccins. Les études que nous avons examinées indiquent également que les patients des professionnels des MAC ont souvent une attitude beaucoup plus négative à l'égard de la vaccination que celle de la population en général. En réalité, l'information sur la vaccination véhiculée par les professionnels de la biomédecine et celle que transmettent les praticiens des MAC sont souvent incompatibles, sinon carrément contradictoires et le non-initié risque de trouver difficile de l'utiliser pour prendre une décision éclairée (Leask, Braunack-Mayer et Kerridge, 2011; Dubé, Vivion, Sauvageau et coll., 2013). Si les recherches existantes laissent entrevoir que le recours aux MAC est clairement lié à une baisse de l'acceptabilité de la vaccination, il faut aussi tenir compte d'autres facteurs comme 
les normes sociales, la perception du risque, les convictions religieuses et l'accessibilité aux services pour mieux comprendre le phénomène de l'hésitation à l'égard de la vaccination. 


\section{BIBLIOGRAPHIE}

Alberta College and Association of Chiropractors. 2015. "Cost and Insurance." Alberta College and Association of Chiropractors. Accessed 4 April 2015, http://www.albertachiro.com/site/cost_and_insurance.

Andrews, G.J., and H. Boon. 2005. "CAM in Canada: Places, Practices, Research." Complementary Therapies in Clinical Practice 11 (1): 21-7. http://dx.doi.org/10.1016/j.ctcp.2004.10.004.

Arsenault, C. 2004. Soins à mon enfant: guide pratique de soins naturels aux enfants. Lebourgneuf, QC: Les Éditions Le Dauphin Blanc.

2009. Accueillir mon enfant naturellement: préconception, grossesse, relevailles, alimentation du bébé, soins naturels au bébé. Lebourgneuf, QC: Les Éditions Le Dauphin Blanc.

Bean, S.J., and J.A. Catania. 2013. "Vaccine Perceptions among Oregon Health Care Providers." Qualitative Health Research 23 (9): 1251-66. http://dx.doi.org/10.1177/1049732313501891.

Birch, Kate. 2015. "Homeoprophylaxis." Vaccine Free: Homeopathic Alternatives to Vaccination (Blog). Accessed 4 April 2015, https://vaccinefree.wordpress.com/homeoprophylaxis/.

Biss, E. 2014. On Immunity: An Inoculation. Minneapolis: Graywolf Press.

Boulianne, N., R. Bradet, D. Audet, M. Ouakki, G. De Serres, M. Guay, E. Dubé, S. Auger, and J. Rivard. 2013. Enquête sur la couverture vaccinale des enfants de 1 an et 2 ans au Québec en 2012. Quebec: Institut national de santé publique du Québec.

Boulianne, N., R. Bradet, D. Audet, M. Ouakki, M. Guay, G. De Serres, J. Rivard, and F. Lavoie. 2011. Enquête sur la couverture vaccinale des enfants de 1 an et 2 ans au Québec en 2010. Quebec: Institut national de santé publique du Québec.

Boyer, R., P. Fisher, L.A. Gallant, J. Hurley, J. Joly, and P. Munk. 2006. "Homeopathy in the Paediatric Population." Paediatrics \& Child Health 11 (2): 76-7.

Busse, J.W., A.V. Kulkarni, J.B. Campbell, and H.S. Injeyan. 2002. "Attitudes toward Vaccination: A Survey of Canadian Chiropractic Students." Canadian Medical Association Journal 166 (12): 1531-4.

Busse, J.W., L. Morgan, and J.B. Campbell. 2005. "Chiropractic Antivaccination Arguments." Journal of Manipulative and Physiological Therapeutics 28 (5): 367-73. http://dx.doi.org/10.1016/j.jmpt.2005.04.011.

Busse, J.W., R. Walji, and K. Wilson. 2011. "Parents' Experiences Discussing Pediatric Vaccination with Healthcare Providers: A Survey of Canadian Naturopathic Patients." PLOS One 6 (8): e22737. http://dx.doi. org/10.1371/journal.pone.0022737.

Busse, J.W., K. Wilson, and J.B. Campbell. 2008. "Attitudes towards Vaccination among Chiropractic and Naturopathic Students." Vaccine 26 (49): 6237-43. http://dx.doi.org/10.1016/j.vaccine.2008.07.020.

Campbell, J.B., J.W. Busse, and H.S. Injeyan. 2000. "Chiropractors and Vaccination: A Historical Perspective." Pediatrics 105 (4): E43. http://dx.doi.org/10.1542/peds.105.4.e43.

Canadian Association of Midwives. 2014. Annual Report 2013-2014. Montreal: Canadian Association of Midwives. Accessed 17 October 2015, http://www.canadianmidwives.org/DATA/TEXTEDOC/Annual-Report2014-FINAL-ENG.pdf.

2015a. "Midwifery Practice - Midwifery Education." The Canadian Association of Midwives. Accessed 4 April 2015, http://www.canadianmidwives.org/midwiferyeducation.html.

2015b. "Midwifery Practice - What is a Midwife?" The Canadian Association of Midwives. Accessed 4 April 2015, http://www.canadianmidwives.org/what-is-amidwife.html. 
Canadian Association of Naturopathic Doctors (CAND). 2004. "Position Paper on Flu Vaccines." Originally published in the CAND Position Papers. January. http://www.zawadahealth.com/catalog/view/ theme/zh/docs/CAND_Position_Paper_Flu_Vaccine.pdf.

2017a. "About Naturopathic Medicine - Guiding Principles." The Canadian Association of Naturopathic Doctors. Accessed 9 January 2017, http://www.cand.ca/guiding-principles/.

2017b. "About Naturopathic Medicine - Naturopathic Medicine Today." The Canadian Association of Naturopathic Doctors. Accessed 9 January 2017, http://www.cand.ca/naturopathic-medicine-today/.

2017c. "About Naturopathic Medicine - What is Naturopathic Medicine?" The Canadian Association of Naturopathic Doctors. Accessed 9 January 2017, http://www.cand.ca/about-naturopathic-medicine/.

2017d. "Common Questions - Naturopathic Visits." The Canadian Association of Naturopathic Doctors. Accessed 9 January 2017, http://www.cand.ca/commonquestions-naturopathic-visits/.

Canadian Chiropractic Association (CCA). 2015a. "Chiropractic Costs \& Coverage." Canadian Chiropractic Association. Accessed 4 April 2015, http://www.chiropractic.ca/about-chiropractic/chiropractic-coverage/.

2015b. "Chiropractic Treatment for Everyone." Canadian Chiropractic Association. Accessed 4 April 2015, http://www.chiropractic.ca/about-chiropractic/how-our-experts

2015c. "Regulation \& Education." Canadian Chiropractic Association. Accessed 4 April 2015, http://www. chiropractic.ca/about-chiropractic/canadian-chiropractors/regulation-and-education/.

2015d. "Vaccination and Immunization." Canadian Chiropractic Association. Accessed 4 April 2015, http:// www.chiropractic.ca/about-cca/code-of-ethics/vaccination-immunization/.

Canadian Medical Protective Association (CMPA). 2008. "New Vaccines - What Are Your Obligations? An Article for Physicians by Physicians." September. Last modified January 2009. https://www.cmpa-acpm.ca/-/new-vaccines-what-are-your-obligations.

Canadian Midwifery Regulators Consortium (CMRC). 2017. "Canadian Model of Midwifery Practice." Canadian Midwifery Regulators Consortium. Accessed 9 January 2017, http://cmrc-ccosf.ca/midwifery-canada.

Canadian Paediatric Society. 2005. "Homeopathy in the Paediatric Population." Paediatrics \& Child Health 10 (3): 173-7.

Canadian Society of Homeopaths. 2015. "Patient Information about Homeopathy" Canadian Society of Homeopaths. Accessed 4 April 2015, http://www.csoh.ca/Homeopathy_Patient_Information.htm.

Cassell, J.A., M. Leach, M.S. Poltorak, C.H. Mercer, A. Iversen, and J.R. Fairhead. 2006. "Is the Cultural Context of MMR Rejection a Key to an Effective Public Health Discourse?" Public Health 120 (9): 783-94. http:// dx.doi.org/10.1016/j.puhe.2006.03.011.

Daley, M.F, N. Liddon, L.A. Crane, B.L. Beaty, J. Barrow, C. Babbel, L.E. Markowitz, E.F. Dunne, S. Stokley, L.M. Dickinson, et al. 2006. "A National Survey of Pediatrician Knowledge and Attitudes Regarding Human Papillomavirus Vaccination." Pediatrics 118 (6): 2280-9. http://dx.doi.org/10.1542/peds.2006-1946.

Dionne, M., N. Boulianne, B. Duval, F. Lavoie, N. Laflamme, J. Carsley, L. Valiquette, S. Gagnon, L. Rochette, and G. De Serres. 2001. "Manque de conviction face à la vaccination chez certains vaccinateurs québécois." Revue canadienne de santé publique 92 (2): 100-4.

Downey, L., P.T. Tyree, C.E. Huebner, and W.E. Lafferty. 2010. "Pediatric Vaccination and Vaccine-Preventable Disease Acquisition: Associations with Care by Complementary and Alternative Medicine Providers." Maternal and Child Health Journal 14 (6): 922-30. http://dx.doi.org/10.1007/s10995-009-0519-5. 
Dubé, E., F. Defay, M. Kiely, C. Sauvageau, V. Gilca, M. Guay, and N. Boulianne. 2011. Connaissances, attitudes et pratiques d'infirmiers(ères), pédiatres et omnipraticiens québécois sur la grippe A(H7N7) et la grippe saisonnière. Quebec, Institut national de santé publique du Québec et ministère de la Santé et des Services sociaux.

Dubé, E., V. Gilca, C. Sauvageau, J.A. Bettinger, F.D. Boucher, S. McNeil, I. Gemmill, F. Lavoie, M. Ouakki, and N. Boulianne. 2012. "Clinicians' Opinions on New Vaccination Programs Implementation." Vaccine 30 (31): 4632-7. http://dx.doi.org/10.1016/j.vaccine.2012.04.100.

Dubé, E., V. Gilca, C. Sauvageau, R. Bradet, F. Lavoie, N. Boulianne, F.D. Boucher, J.A. Bettinger, S. McNeil, and I. Gemmill. 2011. "Acute Otitis Media and Its Prevention by Immunization: A Survey of Canadian Paediatricians' Knowledge, Attitudes and Beliefs." Human Vaccines 7 (4): 429-35. http://dx.doi. org/10.4161/hv.7.4.14141.

Dubé, E., M. Vivion, C. Sauvageau, A. Gagneur, R. Gagnon, and M. Guay. 2013. "How Do Midwives and Physicians Discuss Childhood Vaccination with Parents?" Journal of Clinical Medicine 2 (4): 242-59. http:// dx.doi.org/10.3390/jcm2040242.

2016. "'Nature Does Things Well, Why Should We Interfere?' Vaccine Hesitancy among Mothers." Qualitative Health Research 26 (3): 411-25.

Dubé, E., M. Vivion, A. Valderrama, and C. Sauvageau. 2013. "Attitudes et croyances des sages-femmes québécoises sur la vaccination." "Knowledge and attitudes toward vaccination among midwives in Quebec."] Santé Publique 25 (1): 35-43.

Durand, S., M. Allard, and C. Thibault. 2007. Protéger la population par la vaccination: une contribution essentielle de l'infirmière. Montreal: Ordre des infirmières et infirmiers du Québec.

Ekos Research Associates Inc. 2011. Survey of Parents on Key Issues Related to Immunization. Ottawa: Public Health Agency of Canada.

Ernst, E. 2001. "Rise in Popularity of Complementary and Alternative Medicine: Reasons and Consequences for Vaccination." Vaccine 20 (Suppl 1): S90-3. http://dx.doi.org/10.1016/S0264-410X(01)00290-0.

2011. "Complementary and Alternative Medicine's Opposition to Measles Immunisation Continues." Focus on Alternative and Complementary Therapies 16 (2): 110-14. http://dx.doi.org/10.1111/j.20427166.2011.01092.x.

2015a. "15 'Arguments' for Homeopathy." Edzard Ernst (Blog), 19 March. http://edzardernst. com/2015/03/15-arguments-for-homeopathy/.

2015b. "Homeopathy: Have the Regulators in Ontario Lost Their Senses?" Edzard Ernst (Blog). 25 March. http://edzardernst.com/2015/03/homeopathy-have-theregulators-in-ontario-lost-their-senses/.

Ernst, E., M.H. Cohen, and J. Stone. 2004. "Ethical Problems Arising in Evidence Based Complementary and Alternative Medicine." Journal of Medical Ethics 30 (2): 156-9. http://dx.doi.org/10.1136/ jme.2003.007021.

Fadlon, J. 2005. Negotiating the Holistic Turn: The Domestication of Alternative Medicine. New York: State University of New York.

Flanagan-Klygis, E.A., L. Sharp, and J.E. Frader. 2005. "Dismissing the Family Who Refuses Vaccines: A Study of Pediatrician Attitudes." Archives of Pediatrics \& Adolescent Medicine 159 (10): 929-34. http:// dx.doi.org/10.1001/archpedi.159.10.929.

Gagnon, R. 2012. "L'intégration des sages-femmes d'origine étrangère à la pratique québécoise." In Édiqscope, edited by EDIQ. Quebec: Université Laval, Faculté des lettres.

Gaudino, J.A., and S. Robison. 2012. "Risk Factors Associated with Parents Claiming Personal-Belief Exemptions to School Immunization Requirements: Community and Other Influences on More Skeptical Parents in Oregon, 2006." Vaccine 30 (6): 1132-42. http://dx.doi.org/10.1016/j.vaccine.2011.12.006. 
Gleberzon, B., M. Lameris, C. Schmidt, and J. Ogrady. 2013. "On Vaccination \& Chiropractic: When Ideology, History, Perception, Politics and Jurisprudence Collide." Journal of the Canadian Chiropractic Association 57 (3): 205-13.

Guay, M., F. Gallagher, G. Petit, S. Ménard, P. Clément, and G. Boyer. 2009. Pourquoi les couvertures vaccinales chez les nourrissons de l'Estrie sont-elles sous-optimales? Sherbrooke, QC: Centre de santé et de services sociaux - Institut universitaire de gériatrie de Sherbrooke.

Hall, H.G., L.G. McKenna, and D.L. Griffiths. 2012. "Midwives' Support for Complementary and Alternative Medicine: A Literature Review." Women and Birth; Journal of the Australian College of Midwives 25 (1): 4-12. http://dx.doi.org/10.1016/j.wombi.2010.12.005.

Harris, P.E., K.L. Cooper, C. Relton, and K.J. Thomas. 2012. "Prevalence of Complementary and Alternative Medicine (CAM) Use by the General Population: A Systematic Review and Update." International Journal of Clinical Practice 66 (10): 924-39. http://dx.doi.org/10.1111/j.1742-1241.2012.02945.x.

Health Canada. 2001. Perspectives on Complementary and Alternative Health Care. Ottawa: Health Canada.

2015. "Drugs \& Health Products: Information on Homeopathic Products." Health Canada. Last modified 6 August. http://www.hc-sc.gc.ca/dhp-mps/prodnatur/index-eng.php.

2017. "Drugs \& Health Products: Natural and Non-Prescription Health Products." Health Canada. Accessed 9 January 2017, http://www.hc-sc.gc.ca/dhp-mps/prodnatur/index-eng.php.

Homeopathy Act, Statutes of Ontario 2007, c. 10, Sched. Q. https://www.ontario.ca/laws/statute/07h10

Human Resources and Skills Development Canada. 2017a. "Unit Group - 3122 Chiropractors." Government of Canada. Last modified 23 January. http://noc.esdc.gc.ca/English/noc/ProfileQuickSearch. aspx?val=3\&val1=3122\&ver=16\&val65=chiropractor .

2017b. "Unit Group - 3124 Allied Primary Health Practitioners." Government of Canada. Last modified 23 January. http://noc.esdc.gc.ca/English/noc/ProfileQuickSearch.aspx?ver=16\&val=3\&val1=3124\&v al $65=3$.

2017c. "Unit Group - 3232 Practitioners of Natural Healing." Government of Canada. Last modified 23 January. $\quad$ http://noc.esdc.gc.ca/English/noc/ProfileQuickSearch.aspx?ver=16\&val=3\&val1=3232\&v al $65=3$.

Institute of Medicine (US) Committee on the Use of Complementary and Alternative Medicine by the American Public. 2005. Complementary and Alternative Medicine in the United States. Washington, DC: National Academies Press.

Jones, L., C. Sciamanna, and E. Lehman. 2010. "Are Those Who Use Specific Complementary and Alternative Medicine Therapies Less Likely to be Immunized?" Preventive Medicine 50 (3): 148-54. http://dx.doi.org/10.1016/j.ypmed.2009.12.001.

Kaptchuk, T.J., and D.M. Eisenberg. 2001. "Varieties of Healing. 1: Medical Pluralism in the United States." Annals of Internal Medicine 135 (3): 189-95. http://dx.doi.org/10.7326/0003-4819-135-3-20010807000011.

Kelner, M., B. Wellman, S. Welsh, and H. Boon. 2006. "How Far Can Complementary and Alternative Medicine Go? The Case of Chiropractic and Homeopathy." Social Science \& Medicine 63 (10): 2617-27. http://dx.doi.org/10.1016/j.socscimed.2006.07.005.

Kempe, A., M.M. Patel, M.F. Daley, L.A. Crane, B. Beaty, S. Stokley, J. Barrow, C. Babbel, L.M. Dickinson, J.L. Tempte, et al. 2009. "Adoption of Rotavirus Vaccination by Pediatricians and Family Medicine Physicians in the United States." Pediatrics 124 (5): e809-16. http://dx.doi.org/10.1542/peds.2008-3832.

Kleinman, A. 1997. Writing at the Margin: Discourse between Anthropology and Medicine. Los Angeles: University of California Press. http://dx.doi.org/10.1525/california/9780520209657.001.0001. 
Kriwy, P. 2012. "Similarity of Parents and Physicians in the Decision to Vaccinate Children against Measles, Mumps and Rubella." International Journal of Public Health 57 (2): 333-40. http://dx.doi.org/10.1007/ s00038-011-0326-9.

Lameris, M., C. Schmidt, B. Gleberzon, and J. Ogrady. 2013. "Attitudes toward Vaccination: A Cross-Sectional Survey of Students at the Canadian Memorial Chiropractic College." Journal of the Canadian Chiropractic Association 57 (3): 214-20.

Larson, H., and W. Schulz. 2015. The State of Vaccine Confidence. London: The Vaccine Confidence Project / London School of Hygiene \& Tropical Medicine. http://www.vaccineconfidence.org/The-Stateof-Vaccine-Confidence-2015.pdf.

Leask, J., A. Braunack-Mayer, and I. Kerridge. 2011. "Consent and Public Engagement in an Era of Expanded Childhood Immunisation." Journal of Paediatrics and Child Health 47 (9): 603-7. http://dx.doi. org/10.1111/j.1440-1754.2011.02160.x.

Lee, T., R. Saskin, M. McArthur, and A. McGeer. 2005. "Beliefs and Practices of Ontario Midwives about Influenza Immunization." Vaccine 23 (13): 1574-8. http://dx.doi.org/10.1016/j.vaccine.2004.09.024.

Leib, S., P. Liberatos, and K. Edwards. 2011. "Pediatricians' Experience with and Response to Parental Vaccine Safety Concerns and Vaccine Refusals: A Survey of Connecticut Pediatricians." Public Health Reports 126 (Suppl 2): 13-23.

MacDonald, N., S. MacLeod, M.B. Stanbrook, P.C. Hébert, K. Flegel, and D. Rosenfield. 2011. "No Regulatory Double Standard for Natural Health Products." Canadian Medical Association Journal 183 (18): 2079. http://dx.doi.org/10.1503/cmaj.111739.

Massé, R. 1995. Culture et santé publique. Montreal: Gaëtan Morin éditeur.

McGregor, M., A.A. Puhl, C. Reinhart, H.S. Injeyan, and D. Soave. 2014. "Differentiating Intraprofessional Attitudes toward Paradigms in Health Care Delivery among Chiropractic Factions: Results from a Randomly Sampled Survey." BMC Complementary and Alternative Medicine 14 (1): 51. http://dx.doi. org/10.1186/1472-6882-14-51.

McLeod, S. 2014. "About Homeopathic Nosodes / Homeoprophylaxis." Little Mountain Homeopathy. 19 June. http://www.littlemountainhomeopathy.com/vaccinealternatives.

McMurtry, A., K. Wilson, C. Clarkin, R. Walji, B.C. Kilian, C.C. Kilian, L. Lohfeld, B. Alolabi, C. Hagino, and J.W. Busse. 2015. "The Development of Vaccination Perspectives among Chiropractic, Naturopathic and Medical Students: A Case Study of Professional Enculturation." Advances in Health Sciences Education: Theory and Practice 20 (5): 1291-302.

Medd, E.A., and M.L. Russell. 2009. "Personal and Professional Immunization Behavior among Alberta Chiropractors: A Secondary Analysis of Cross-Sectional Survey Data." Journal of Manipulative and Physiological Therapeutics 32 (6): 448-52. http://dx.doi.org/10.1016/j.jmpt.2009.06.006

National Center for Complementary and Integrative Health (NIH). 2016. "Complementary, Alternative, or Integrative Health: What's In a Name?" National Center for Complementary and Integrative Health. June. https://nccih.nih.gov/health/whatiscam.

National Health and Medical Research Council (NHMRC). 2015a. NHMRC Information Paper: Evidence on Effectiveness of Homeopathy for Treating Health Conditions. Canberra: National Health and Medical Research Council.

2015b. "NHMRC Statement: Statement on Homeopathy." Australian Government - National Health and Medical Research Council. March. https://www.nhmrc.gov.au/files_nhmrc/publications/attachments/cam02_nhmrc_statement_homeopathy.pdf?.

National United Professional Association of Trained Homeopaths (NUPATH). 2015. NUPATH. Accessed 4 April 2015, http://www.nupath.org. 
Ning, A.M. 2013. "How 'Alternative' is CAM? Rethinking Conventional Dichotomies between Biomedicine and Complementary/Alternative Medicine." Health 17 (2): 135-58. http://dx.doi. org/10.1177/1363459312447252.

Opel, D.J., J.D. Robinson, J. Heritage, C. Korfiatis, J.A. Taylor, and R. Mangione-Smith. 2012. "Characterizing Providers' Immunization Communication Practices during Health Supervision Visits with Vaccine-Hesitant Parents: A Pilot Study." Vaccine 30 (7): 1269-75. http://dx.doi.org/10.1016/j.vaccine.2011.12.129.

Page, S.A., M.L. Russell, M.J. Verhoef, and H.S. Injeyan. 2006. "Immunization and the Chiropractor-Patient Interaction: A Western Canadian Study." Journal of Manipulative and Physiological Therapeutics 29 (2): 156-61. http://dx.doi.org/10.1016/j.jmpt.2005.12.005.

Park, J. 2005. "Use of Alternative Health Care." Health Reports 16 (2): 39-42.

Pélissier-Simard, L., and M. Xhignesse. 2008. "Les approches complémentaires en santé." Le Médecin du Québec 43 (1): 23-30.

Picard, A. 2015. "We're Aiding and Abetting Homeopathic Quackery." Globe and Mail. 31 March.

Posfay-Barbe, K.M., U. Heininger, C. Aebi, D. Desgrandchamps, B. Vaudaux, and C.A. Siegrist. 2005. "How Do Physicians Immunize Their Own Children? Differences among Pediatricians and Nonpediatricians." Pediatrics 116 (5): 623-33. http://dx.doi.org/10.1542/peds.2005-0885.

Pray, W.S. 1996. "The Challenge to Professionalism Presented by Homeopathy." American Journal of Pharmaceutical Education 60 (2): 198-204.

Puhl, A.A., C.J. Reinhart, J.B. Doan, M. McGregor, and H.S. Injeyan. 2014. "Relationship between Chiropractic Teaching Institutions and Practice Characteristics among Canadian Doctors of Chiropractic: A Random Sample Survey." Journal of Manipulative and Physiological Therapeutics 37 (9): 709-18. http://dx.doi.org/10.1016/j.jmpt.2014.09.005.

Russell, M.L., H.S. Injeyan, M.J. Verhoef, and M. Eliasziw. 2004. "Beliefs and Behaviours: Understanding Chiropractors and Immunization." Vaccine 23 (3): 372-9. http://dx.doi.org/10.1016/j.vaccine.2004.05.027.

Russell, M. L., M.J. Verhoef, and H.S. Injeyan. 2005. "Are Chiropractors Interested in Participating in Immunization Awareness and Promotion Activities?" Revue canadienne de santé publique 96 (3): 194-6.

Salmon, D.A., L.H. Moulton, S.B. Omer, M.P. DeHart, S. Stokley, and N.A. Halsey. 2005. "Factors Associated with Refusal of Childhood Vaccines among Parents of School-Aged Children: A Case-Control Study." Archives of Pediatrics \& Adolescent Medicine 159 (5): 470-6. http://dx.doi.org/10.1001/archpedi.159.5.470.

Shang, A., K. Huwiler-Muntener, L. Nartey, P. Juni, S. Dorig, J.A. Sterne, D. Pewsner, and M. Egger. 2005. "Are the Clinical Effects of Homoeopathy Placebo Effects? Comparative Study of Placebo-Controlled Trials of Homoeopathy and Allopathy." The Lancet 366 (9487): 726-32. http://dx.doi.org/10.1016/S01406736(05)67177-2.

Shelley, J., M. Clark, and T. Caulfield. 2015. "The Face of Chiropractic: Evidence-Based?" Focus on Alternative and Complementary Therapies 20 (1): 13-22.

Smith, P.J., S.G. Humiston, E.K. Marcuse, Z. Zhao, C.G. Dorell, C. Howes, and B. Hibbs. 2011. "Parental Delay or Refusal of Vaccine Doses, Childhood Vaccination Coverage at 24 Months of Age, and the Health Belief Model." Public Health Reports 126 (Suppl 2): 135-46.

Tataryn, D.J. 2002. "Paradigms of Health and Disease: A Framework for Classifying and Understanding Complementary and Alternative Medicine." Journal of Alternative and Complementary Medicine (New York, N.Y.) 8 (6): 877-92. http://dx.doi.org/10.1089/10755530260511874.

Vivion, M., A. Valderrama, D. Audet, and C. Sauvageau. 2011. "Vaccination et pratique sage-femme au Québec." Paper presented at 15s Journées annuelles de santé publique (JASP), Montreal, QC, 28 November-1 December. 
Weeks, C. 2015a. "Health Experts Criticize Government Approval of Homeopathic 'Vaccines'." Globe and Mail. 18 February.

2015b. "Health Experts Question Lack of Crackdown on 'Homeopathic Vaccines'." Globe and Mail. 17 February.

"What Mothers Say: The Canadian Maternity Experiences Survey." 2009. Public Health Agency of Canada. 25 March. http://www.phac-aspc.gc.ca/rhs-ssg/survey-enquete/mes-eem-eng.php.

Wieland, L.S., E. Manheimer, and B.M. Berman. 2011. "Development and Classification of an Operational Definition of Complementary and Alternative Medicine for the Cochrane Collaboration." Alternative Therapies in Health and Medicine 17 (2): 50-9.

Williams, S.E. 2014. "What are the Factors that Contribute to Parental Vaccine-Hesitancy and What Can We Do about It?" Human Vaccines \& Immunotherapeutics 10 (9): 2584-96. http://dx.doi.org/10.4161/ hv.28596.

Wilson, K., E. Mills, H. Boon, G. Tomlinson, and P. Ritvo. 2004. "A Survey of Attitudes towards Paediatric Vaccinations amongst Canadian Naturopathic Students." Vaccine 22 (3-4): 329-34. http://dx.doi. org/10.1016/j.vaccine.2003.08.014.

Wilson, K., J.W. Busse, A. Gilchrist, S. Vohra, H. Boon, and E. Mills. 2005a. "Characteristics of Pediatric and Adolescent Patients Attending a Naturopathic College Clinic in Canada." Pediatrics 115 (3): e338-43. http://dx.doi.org/10.1542/peds.2004-1901.

Wilson, K., E. Mills, G. Norman, and G. Tomlinson. 2005b. "Changing Attitudes towards Polio Vaccination: A Randomized Trial of an Evidence-Based Presentation versus a Presentation from a Polio Survivor." Vaccine 23 (23): 3010-15. http://dx.doi.org/10.1016/j.vaccine.2004.12.002.

Yaqub, O., S. Castle-Clarke, N. Sevdalis, and J. Chataway. 2014. "Attitudes to Vaccination: A Critical Review." Social Science \& Medicine 112: 1-11. http://dx.doi.org/10.1016/j.socscimed.2014.04.018.

Zhang, J., A. Ohinmaa, T.-H. Nguyen, L. Mashinter, M.N. Hanrahan, J. Loewen, W. Vaudry, and P. Jacobs. 2008. "Determinants for Immunization Coverage by Age 2 in a Population Cohort in the Capital Health Region, Edmonton, Alberta." Canada Communicable Disease Report 34 (9): 1-11.

Zuzak, T.J., I. Zuzak-Siegrist, L. Rist, G. Staubli, and A.P. Simoes-Wust. 2008. "Attitudes towards Vaccination: Users of Complementary and Alternative Medicine versus Non-Users." Swiss Medical Weekly 138 (47-8): 713-18. http://dx.doi.org/2008/47/smw-12423. 